\title{
S-Benzoxazolyl (SBox) as a Stable Protecting Moiety and a Potent Anomeric Leaving Group in Oligosaccharide Synthesis
}

\author{
Medha N. Kamat, Cristina De Meo, and Alexei V. Demchenko \\ Department of Chemistry and Biochemistry, University of Missouri - St. Louis, One University \\ Boulevard, St. Louis, Missouri 63121, USA; e-mail demchenkoa@umsl.edu
}

\begin{abstract}
As a part of a program for developing new versatile building blocks for stereoselective glycosylation and convergent oligosaccharide synthesis, we demonstrated that $S$-benzoxazolyl (SBox) glycosides are stable toward major protecting group manipulations employed in carbohydrate chemistry. On the other hand, they can be glycosidated under relatively mild reaction conditions to afford either 1,2trans or 1,2-cis-linked disaccharides. Selective and chemoselective activations of the SBox moiety were also proved to be feasible, which was demonstrated by synthesizing a number of oligosaccharide sequences.
\end{abstract}

\section{Introduction}

Glycosyl thioimidates, compounds bearing $\mathrm{SCR}^{1}=\mathrm{NR}^{2}$ aglycone, have been known for decades, yet their synthetic value as versatile intermediates in carbohydrate chemistry has only recently come to the fore. ${ }^{1}$ Relatively low stability of the previously studied thioimidates was the major reason the use of benzothiazolyl, ${ }^{2}$ pyridin-2-yl, ${ }^{3-5}$ pyrimidin-2-yl, ${ }^{3,6}$ imidazolin-2$\mathrm{yl},{ }^{3}$ and $1^{\prime}$-phenyl-1H-tetrazolyl ${ }^{7}$ glycosides in oligosaccharide synthesis was limited. Our laboratory has been primarily investigating a family of substituted oxazol(in)es and thiazol(in) es as complimentary glycosyl donors for chemical glycosylation. ${ }^{8}$ We already demonstrated that this class of compounds can serve as excellent intermediates in stereoselective glycosylations $9-11$ and convergent oligosaccharide syntheses via conceptually novel strategies. ${ }^{12-15}$ Another important recent development in this area is the anomeric phosphorylation of glycosyl thioimidates. ${ }^{16,17}$

Side-by-side comparison of an array of structurally related cyclic thioimidates ${ }^{18}$ revealed that 1-S-benzoxazolyl (SBox) derivatives bear major positive traits of a modern glycosyl donor: accessibility, odorless preparation, stability toward many reaction conditions employed in carbohydrate chemistry, mild and selective activation for glycosylation, and excellent stereoselectivity. In the preceding article ${ }^{19}$ we demonstrated that the SBox glycosides 1-9 (Figure 1) can be successfully prepared from a variety of synthetic precursors and can be applied as glycosyl donors for stereoselective glycosylation. Herein we present our thorough evaluation of the SBox derivatives in glycoside synthesis and their application to the synthesis of a variety of compounds ranging from relatively simple partially protected glycosyl acceptors to complex oligosaccharide sequences. 


\section{Results and Discussion}

In the preceding article, we established the activation pathways and experimental conditions for the glycosidation of SBox glycosides $;{ }^{19}$ herein, we turn our attention to investigating their properties as glycosyl donors in the formation of various glycosidic linkages. A unique feature of the SBox glycosyl donors is that a broad range of conceptually different approaches can be applied to their glycosidation. The reaction conditions range from traditional thioglycoside activators: MeOTf and NIS/TfOH to mildly electrophilic metal salts, such as AgOTf and $\mathrm{Cu}$ $(\mathrm{OTf})_{2}$. In addition, protic and Lewis acids were also shown as promoters for this class of compounds, although at this stage the effectiveness of this type of activation is modest.

\section{Glycosidation of the SBox derivatives: Synthesis of 1,2-trans glycosides}

As previously reported,${ }^{19}$ per-acetylated SBox glycosides (such as 1) provide somewhat compromised results due to competing acetyl migration from the $\mathrm{O}-2$ of glycosyl donor to the free hydroxyl of the glycosyl acceptor. ${ }^{19}$ Hence, our main effort for the synthesis of 1,2-translinked glycosides has been focused on the investigation of the per-benzoylated SBox glycosides (2-4). Also, having already ascertained that the per-benzoylated SBox glycosides could be efficiently activated with AgOTf or MeOTf, our main effort has been directed on the application of these two promoters. Herein we report that per-benzoylated SBox glycosyl donors of the D-gluco, D-galacto-, and D-manno series $(\mathbf{2}, \mathbf{3}$, and $\mathbf{4}$, respectively) are very efficient glycosyl donors for the synthesis of 1,2-trans linked disaccharides. As highlighted in Table 1, reactions with differently protected glycosyl acceptors 10, 12, 14, 16, 18, and 20 of the D-gluco and D-galacto series gave the corresponding disaccharides 11, 13, 15, 17, 19, 2123 in high yields of $86-95 \%$ and complete stereoselectivity. The complete 1,2-trans stereoselectivity is attributed to the assistance of a participating substituent at the C-2 position.

\section{Glycosidation of the SBox derivatives: Synthesis of 1,2-cis glycosides}

Stereoselective synthesis of 1,2-cis glycosides from SBox glycosyl donors was also investigated. In this case, we investigated glycosyl donors bearing a non-participating substituent at C-2, per-benzylated SBox glycoside 5 or its 2- $O$-benzyl-3,4,6-tri- $O$-acetyl counterpart 7. Similarly, glycosidations of the SBox derivatives of the D-manno (3) and Dgalacto series $(\mathbf{4}$ or $\mathbf{8})$ respectively, were probed. For the highly reactive per-benzylated glycosyl donors 5 or $6, \mathrm{Cu}(\mathrm{OTf})_{2}$ was found to be the promoter of choice. Although reactions performed in 1,2-DCE were found to be high yielding, the stereoselectivity was below average (typically $\alpha / \beta 2 / 1$ ) in the majority of cases. While no improvement in stereoselectivity was achieved by changing promoters, significantly higher stereoselectivity was accomplished when the glycosylation was performed in toluene-dioxane $(1 / 3, \mathrm{v} / \mathrm{v})$, a participating solvent mixture. 20 As a result of varying the reaction solvent, significantly improved stereoselectivity could be achieved (up to $\alpha / \beta 7 / 1$, Entries 1-5, Table 2).

Application of partially acetylated glycosyl donors $\mathbf{7}$ or $\mathbf{8}$ was found to be especially beneficial for 1,2-cis glycosylation. In a number of cases, very high or even complete 1,2-cis stereoselectivity was achieved even in dichloromethane, a solvent that does not usually favor 1,2-cis glycosylation (see Entries 6-14, Table 2). Arguably, the stereoselectivity achieved herein favorably compares with the most selective procedures for direct 1,2-cis glycosylation developed to date. ${ }^{21,22}$ It should be noted that in spite of significant improvements that have emerged in the past decade, stereocontrolled synthesis of 1,2-cis glycosides still remains a significant challenge.

\section{Stability of the 1-SBox Glycosides: Synthesis of Glycosyl Acceptors}

A significant drawback of many classes of glycosyl donors is their poor stability toward protecting group manipulations. For instance, labile glycosyl donors such as bromides, 23,24 
trichloroacetimidates, ${ }^{25}$ phosphites, ${ }^{26}$ phosphates, ${ }^{27}$ etc. should be obtained directly prior to the glycosylation. In this respect, stable glycosyl donors, such as fluorides, ${ }^{28,29}$ alkyl or aryl thioglycosides, ${ }^{30,31} \mathrm{O}$-alkenyl glycosides, ${ }^{32,33}$ or selenoglycosides, ${ }^{34}$ offer a significant advantage in that a stable leaving group can also serve as a temporary anomeric protecting group. This would allow the installation of a required protecting group pattern and preclude additional manipulations at the anomeric center prior to glycosylation. The evaluation of the compatibility of SBox glycosides with reaction conditions required for installing or removing common classes of protecting groups seemed to be a logical step in the systematic study of these novel derivatives.

It should be noted that the majority of SBox derivatives investigated in our laboratory were found to be stable crystalline compounds that could be stored at ambient temperature and humidity. ${ }^{19}$ As to the chemical stability of the SBox glycosides, we performed a number of experiments that demonstrated the relatively high stability of the SBox moiety. Thus, preliminary experiments with SBox derivative $\mathbf{7}$ demonstrated that this compound could be efficiently deacetylated under conventional Zemplen conditions. ${ }^{35}$ The intermediate $\mathbf{4 1}$ was found to be stable under standard reaction conditions for the introduction of alkyl, acyl, and acetal substituents (Scheme 1). ${ }^{36}$ As a result, we accomplished the syntheses of a range of differently protected building blocks $\mathbf{5}, \mathbf{4 2}-\mathbf{4 4}$ containing the SBox anomeric moiety.

However, when Zemplen conditions (catalytic $\mathrm{MeONa}$ in $\mathrm{MeOH}$ ) were applied to the deprotection of the tetraacetyl derivative $\mathbf{1}$ or its triacetylated counterpart $\mathbf{9}$, only traces of the expected intermediate $\mathbf{4 5}$ were detected in the reaction mixture. Disappointingly, methyl Dglucopyranoside $(\alpha / \beta=1.3 / 1)$ was found to be the major product of this reaction - an indication of the anomeric leaving group displacement. Taking into consideration that deacetylation of the 2-benzyl derivative $\mathbf{7}$ proceeded in a nearly quantitative yield (see the synthesis of $\mathbf{4 1}$, Scheme 1), the results with compounds 1 or 9 were rather surprising. To overcome this problem, the deacetylation of $\mathbf{1}$ was performed using a saturated solution of ammonia in $\mathrm{MeOH}(\mathrm{pH} \leq$ 8). Simple precipitation from the reaction mixture afforded the desired reaction intermediate 45, which was found to be compatible with conventional tritylation, benzoylation, and detritylation conditions. As a result, derivatives $\mathbf{2}$ and $\mathbf{4 6}$ were obtained in good overall yields (Scheme 2).

However, when the intermediate $\mathbf{4 5}$ was subjected to strongly basic reaction conditions $(\mathrm{BnBr}$ and $\mathrm{NaH}$ in DMF, see the attempted synthesis of $\mathbf{5}$, Scheme 2), departure of the SBox moiety resulted in the formation of D-glucose, which was subsequently benzylated. It should be reminded that per-benzylated SBox derivative $\mathbf{5}$ could be readily obtained from the 2-benzyl SBox intermediate $\mathbf{4 1}$ under the same reaction conditions in $90 \%$ yield. The disparity of results obtained from 2-benzyl $(\mathbf{7} \rightarrow \mathbf{4 1} \rightarrow \mathbf{5})$ and 2-acyl/hydroxyl derivatives $(\mathbf{1} \rightarrow \mathbf{4 5} \rightarrow \mathbf{5})$ made us believe that while the SBox moiety itself is stable toward strong bases ( $\mathrm{MeONa}, \mathrm{NaH}, \mathrm{NaOH}$, see for example the syntheses of $\mathbf{5}$ and $\mathbf{4 1}$, Scheme 1), it readily departs upon the nucleophilic attack of the deprotonated C-2 hydroxyl on the anomeric center (see the proposed reaction intermediate A, Scheme 2).

\section{SBox glycosides as building blocks in convergent oligosaccharide synthesis}

An important feature of a glycosyl donor would be its applicability to multistep oligosaccharide synthesis via convergent building block-based pathways. ${ }^{37}$ Having completed the stereoselectivity studies (see Tables 1 and 2), we wanted to investigate whether the SBox glycosides could be chemoselectively activated in accordance with the armed-disarmed approach developed by Fraser-Reid. 38,39 According to this strategy, a significantly more reactive (armed) benzylated glycosyl donor can be chemoselectively activated in the presence of the acylated (disarmed) derivative to afford a disaccharide. We found that the SBox glycosides also follow general chemoselectivity principle. Thus, armed SBox glycoside donor 
5 could be activated over electronically disarmed SBox glycosyl acceptor $\mathbf{4 6}$ in the presence of $\mathrm{Cu}(\mathrm{OTf})_{2}$ (Scheme 3). Therefore obtained disaccharide $\mathbf{4 7}$ bearing the SBox leaving group can then be coupled with a suitable acceptor under typical reaction conditions for the SBox activation.

Our other aim was to determine whether the SBox derivatives could be coupled with glycosyl acceptors containing other types of anomeric leaving groups. The key requirement for such activation would be the availability of a promoter that could selectively activate the SBox moiety over a stable anomeric moiety of the glycosyl acceptor, such as $S$-ethyl. Our initial assumption was that such selective activation could be accomplished using AgOTf as a promoter. Thus, in studies with glycosyl donors 2 and 7, we chose $S$-ethyl glycoside 49 as glycosyl acceptor (Scheme 4). Remarkably, the glycosylations involving selective activation of the SBox leaving group afforded the corresponding disaccharide products $\mathbf{5 0}$, and $\mathbf{5 2}$ with excellent stereoselectivity and yields of 98-99\%. Very importantly, no side products involving self-condensation of the glycosyl acceptor were detected. Similarly, the SBox moiety could be activated in the presence of the $O$-pentenyl moiety. ${ }^{11}$ Furthermore, in an independent study of the STaz glycosidation protocol, it was also established that the SBox moiety can be selectively activated over the STaz anomeric moiety using $\mathrm{Cu}(\mathrm{OTf})_{2}$ as a promoter. ${ }^{9}$

The key feature of the oligosaccharide synthesis via selective activation is that the disaccharides obtained can be immediately used in subsequent glycosidation. In the case of the disaccharides $\mathbf{5 0}$ and 52, the second step activation should be feasible in the presence of NIS/TfOH or other suitable activators for $S$-alkyl/aryl moieties. ${ }^{30-32}$ Additionally, selective activation of S-ethyl over the O-pentenyl moiety can be achieved in the presence of MeOTf in accordance with the semi-orthogonal strategy developed in our laboratory. ${ }^{40}$ To explore this possibility, we performed the coupling of the $S$-ethyl disaccharide donors $\mathbf{5 0}$ or $\mathbf{5 2}$ with glycosyl acceptors 48 or 53 in the presence of MeOTf to afford the corresponding trisaccharide derivatives 51 or $\mathbf{5 4}$ in 92 or $90 \%$ yield, respectively (Scheme 4). The $O$-pentenyl moiety of the trisaccharide donor 54 was then activated for the reaction with glycosyl acceptor 14 in the presence of NIS/ $\mathrm{TfOH}$. As a result, a tetrasaccharide derivative $\mathbf{5 5}$ was isolated in 73\% yield. These examples serve as a clear illustration of the enhanced ability to obtain oligosaccharides via sequential activation in a convergent fashion with no additional protecting/leaving group manipulations between the glycosylation steps.

\section{Conclusions}

Based on the results presented, we conclude that the SBox glycosides are stable toward majority of protecting group manipulations employed in carbohydrate chemistry. It has been demonstrated that the SBox moiety is even stable toward strong bases if the O-2 position of the pyranose ring is protected with a stable moiety, such as benzyl. When the protection is removed, stability of the SBox moiety significantly decreases. The SBox glycosides were also found to be suitable building blocks for chemoselective armed-disarmed activations and for selective sequential activations over other classes of leaving groups.

\section{Experimental Part}

For general procedures for the preparation of di- and oligosaccharides, refer to the preceding article: Method A - MeOTf, Method B - AgOTf, Method C - Cu(OTf) ${ }_{2 \cdot}{ }^{19}$

Methyl 2,3,4-tri- $O$-benzoyl-6- $O$-(2,3,4,6-tetra- $O$-benzoyl- $\beta$-D-glucopyranosyl)- $\beta$-Dgalactopyranoside (11) was obtained using Method A from $\mathbf{2}$ and $\mathbf{1 0}$ in $92 \%$ yield. Analytical data for $\mathbf{1 1}$ were essentially the same as reported previously. 41 
6- $O$-(2,3,4,6-Tetra- $O$-benzoyl- $\beta$-D-glucopyranosyl)-1,2:3,4-di- $O$-isopropylidene- $\alpha$-Dgalactopyranose (13) was obtained from $\mathbf{2}$ and $\mathbf{1 2}$ using Method B in $91 \%$ or by Method C in $70 \%$ yield. Analytical data for $\mathbf{1 3}$ were essentially the same as reported previously. ${ }^{9}$

Methyl 6- $O$-(2,3,4,6-tetra- $O$-benzoyl- $\beta$-D-glucopyranosyl)-2,3,4-tri- $O$-benzyl- $\alpha$-Dglucopyranoside (15) was obtained using Method A, from $\mathbf{2}$ and $\mathbf{1 4} 42$ in $95 \%$ yield. Analytical data for $\mathbf{1 5}$ were essentially the same as reported previously. 43

2-Trimethylsilylethyl 2-O-(2,3,4,6-Tetra- $O$-benzoyl- $\beta$-D-glucopyranosyl)-3-Obenzyl-4,6- $\boldsymbol{O}$-benzylidene- $\boldsymbol{\beta}$-D-galactopyranoside (17) was obtained using Method A from 2 and $\mathbf{1 6} \mathbf{4}^{44}$ in $94 \%$ yield. Analytical data for $\mathbf{1 7}: \mathrm{R}_{\mathrm{f}}=0.37$ (ethyl acetate - hexane, 3/7, v/v); $[\alpha]_{\mathrm{D}}{ }^{22} 39.9^{\circ}\left(\mathrm{c}=1.0, \mathrm{CHCl}_{3}\right) ;{ }^{1} \mathrm{H}$-n.m.r.: $\delta, 0.00\left(\mathrm{~s}, 9 \mathrm{H}, \mathrm{SiCH}_{3}\right), 1.01(\mathrm{t}, 2 \mathrm{H}, \mathrm{J}=8.7 \mathrm{~Hz}$, $\left.\mathrm{CH}_{2} \mathrm{TMS}\right), 3.40\left(\mathrm{dd}, 1 \mathrm{H}, \mathrm{J}_{3,4}=3.6 \mathrm{~Hz}, \mathrm{H}-3\right), 3.63\left(\mathrm{~m}, 1 \mathrm{H}, \mathrm{CH}_{2}{ }^{\mathrm{a}}\right), 3.80(\mathrm{dd}, 1 \mathrm{H}, \mathrm{H}-4), 3.87$ $\left(\mathrm{dd}, 1 \mathrm{H}, \mathrm{J}_{5,6 \mathrm{a}}=2.7, \mathrm{~J}_{6 \mathrm{a}, 6 \mathrm{~b}}=12.4, \mathrm{H}-6 \mathrm{a}\right), 3.97-4.27\left(\mathrm{~m}, 5 \mathrm{H}, \mathrm{CH}_{2}{ }^{\mathrm{b}}, \mathrm{H}-2^{\prime}, 5,5^{\prime}, 6 \mathrm{~b}\right), 4.35(\mathrm{dd}$, $2 \mathrm{H}, \mathrm{J}^{2}=12.8 \mathrm{~Hz}, \mathrm{CH}_{2} \mathrm{Ph}$ ), $4.42\left(\mathrm{~d}, 1 \mathrm{H}, \mathrm{J}_{1,2}=7.7 \mathrm{~Hz}, \mathrm{H}-1\right), 4.50\left(\mathrm{dd}, 1 \mathrm{H}, \mathrm{H}-6 \mathrm{~b}^{\prime}\right), 4.65$ (dd, $1 \mathrm{H}$, $\left.\mathrm{J}_{5^{\prime}, 6 \mathrm{a}^{\prime}}=3.8 \mathrm{~Hz}, \mathrm{~J}_{6 \mathrm{a}^{\prime}, 6 \mathrm{~b}^{\prime}}=12.6, \mathrm{H}-6 \mathrm{a}^{\prime}\right), 5.25(\mathrm{~s}, 1 \mathrm{H}, \mathrm{CHPh}), 5.39\left(\mathrm{~d}, 1 \mathrm{H}, \mathrm{J}_{1^{\prime}, 2^{\prime}}=7.9 \mathrm{~Hz}, \mathrm{H}-1^{\prime}\right)$, $5.65\left(\mathrm{dd}, 1 \mathrm{H}, \mathrm{J}_{2^{\prime}, 3^{\prime}}=8.7 \mathrm{~Hz}, \mathrm{H}-2^{\prime}\right), 5.79\left(\mathrm{dd}, 1 \mathrm{H}, \mathrm{J}_{4^{\prime}, 5^{\prime}}=9.4 \mathrm{~Hz}, \mathrm{H}-4^{\prime}\right), 5.86\left(\mathrm{dd}, 1 \mathrm{H}, \mathrm{J}_{3^{\prime}, 4^{\prime}}=9.5\right.$ Hz, H-3') ppm; i ${ }^{13}$ C-n.m.r.: $\delta, ~ 55.4,60.6,62.9,66.8,67.1,68.8,69.1,70.1,70.2,70.5,73.6$, $75.2,75.9,76.3,77.4,77.9,79.1,80.4,82.3,97.9,98.1,127.8(\times 3), 128.0,128.1(\times 3), 128.3$ $(\times 2), 128.5(\times 2), 128.6(\times 7), 128.7(\times 5), 128.8(\times 2), 129.2,129.3,129.5,129.9(\times 4), 130.0$ $(\times 2), 130.1,133.2,133.3,133.6,133.9,134.3,138.4(\times 2), 138.9$ ppm; HR-FAB MS [M + $\mathrm{Na}]^{+}$calcd for $\mathrm{C}_{59} \mathrm{H}_{60} \mathrm{NaO}_{15} \mathrm{Si} 1059.3579$, found 1059.3589 .

Methyl 3- $O$-(2,3,4,6-tetra- $O$-benzoyl- $\beta$-D-glucopyranosyl)-2- $O$-benzyl-4,6- $O$ benzylidene- $\boldsymbol{\alpha}$-D-glucopyranoside (19) was obtained using Method A from $\mathbf{2}$ and $18^{45}$ in $86 \%$ yield. Analytical data for $\mathbf{1 9}$ were essentially the same as reported previously. 46

Methyl 4- $O$-(2,3,4,6-tetra- $O$-benzoyl- $\beta$-D-glucopyranosyl)-2,3,6-tri- $O$-benzyl- $\alpha$-Dglucopyranoside (21) was obtained using Method A from 2 and $\mathbf{2 0}^{47}$ in $86 \%$ yield. Analytical data for $\mathbf{2 1}$ were essentially the same as reported previously. ${ }^{9}$

Methyl 6- $O$-(2,3,4,6-tetra- $O$-benzoyl- $\beta$-D-galactopyranosyl)-2,3,4-tri- $O$-benzyl- $\alpha$-Dglucopyranoside (22) was obtained using Method B from 3 and 14 in $92 \%$ yield. Analytical data for $\mathbf{2 2}$ were essentially the same as reported previously. 48

Methyl 6- $O$-(2,3,4,6-tetra- $O$-benzoyl- $\alpha$-D-mannopyranosyl)-2,3,4-tri- $O$-benzyl- $\alpha$-Dglucopyranoside (23) was obtained using Method A from 4 and $\mathbf{1 4}$ in $92 \%$ yield Analytical data for 23 were essentially same as reported previously. ${ }^{9}$

Methyl 2,3,4-tri- $O$-benzyl-6- $O$-(2,3,4,6-tetra- $O$-benzyl-D-glucopyranosyl)- $\alpha$-Dglucopyranoside (24) was obtained using Method $C$ from 5 and $\mathbf{1 4}$ in toluene - dioxane (1/3, $\mathrm{v} / \mathrm{v}, 1 \mathrm{~mL})$ in $95 \%$ yield $(\alpha / \beta=6 / 1)$. Analytical data for $\mathbf{2 4}$ were essentially the same as reported previously. 49

6- $O$-(2,3,4,6-Tetra- $O$-benzyl-D-glucopyranosyl)-1,2:3,4-di- $O$-isopropylidene- $\alpha$-Dgalactopyranose (25) was obtained using Method C from 5 and 12 in toluene - dioxane ( $1 \mathrm{~mL}$, $3 / 1, \mathrm{v} / \mathrm{v})$ in $89 \%$ yield $(\alpha / \beta=5.4 / 1)$. Analytical data for 25 were essentially the same as reported previously. 50

Methyl 2- $O$-benzyl-3- $O$-(2,3,4,6-tetra- $O$-benzyl-D-glucopyranosyl)-4,6- $O$-benzylidenea-D-glucopyranoside (26) was obtained using Method $\mathrm{C}$ from 5 and $\mathbf{1 8}$ in toluene - dioxane $(1: 3, \mathrm{v} / \mathrm{v}, 1 \mathrm{~mL})$ in $65 \%(\alpha / \beta=4 / 1)$ or in $1,2-\mathrm{DCE}$ in $89 \%(\alpha / \beta=3 / 1)$ yield. Analytical data for 26 were essentially the same as reported previously. 51 
Methyl 2-O-(2,3,4,6-tetra- $O$-benzyl-D-glucopyranosyl)-3,4,6-tri- $O$-benzyl- $\alpha$-Dglucopyranoside (28) was obtained using Method $C$ from $\mathbf{5}$ and $27^{52}$ in toluene - dioxane $(1 / 3, \mathrm{v} / \mathrm{v}, 1 \mathrm{~mL})$ in $68 \%(\alpha / \beta=7 / 1)$ or in $1,2-\mathrm{DCE}$ in $61 \%(\alpha / \beta=2 / 1)$ yield. Analytical data for $\alpha-\mathbf{2 8}$ were essentially the same as reported previously. ${ }^{9}$

Methyl 2,3,6-tri- $O$-benzyl-4- $O$-(2,3,4,6-tetra- $O$-benzyl-D-glucopyranosyl)- $\alpha$-Dglucopyranoside (29) was obtained using Method $C$ from 5 and 20 in toluene - dioxane (1/3, $\mathrm{v} / \mathrm{v}, 1 \mathrm{~mL})$ in $67 \%(\alpha / \beta=3 / 1)$ or in $1,2-\mathrm{DCE}$ in $41 \%(\alpha / \beta=1.5 / 1)$ yield. Analytical data for 29 were essentially the same as reported previously. 53

Methyl 2,3,6-tri- $O$-benzyl-4- $O$-(2,3,4,6-tetra- $O$-benzyl-D-mannopyranosyl)- $\alpha$-Dglucopyranoside (30) was obtained using Method A from 6 and 20 in $83 \%$ yield $(\alpha / \beta=1 / 5.9)$. Analytical data for $\mathbf{3 0}$ were essentially the same as reported previously. 54

Methyl 2,3,4-tri- $O$-benzoyl-6- $O$-(2,3,4,6-tetra- $O$-benzyl-D-mannopyranosyl)- $\alpha$-Dglucopyranoside (32) was obtained using Method A from 6 and $\mathbf{3 1}$ in $72 \%$ yield $(\alpha / \beta=1 / 2.2)$. Analytical data for $\mathbf{3 2}$ were essentially the same as reported previously. 54

Methyl 2- $O$-benzyl-3- $O$-(2,3,4,6-tetra- $O$-benzyl-D-mannopyranosyl)-4,6- $O$-benzylidene$\alpha$-D-glucopyranoside (33) was obtained using Method A from 6 and 18 in $83 \%$ yield $(\alpha / \beta=$ $1 / 2.5$ ). Analytical data for 33 were essentially the same as reported previously. 51

6-O-(3,4,6-Tri- $O$-acetyl-2- $O$-benzyl-D-glucopyranosyl)-1,2:3,4-di- $O$-isopropylidene- $\alpha$ D-galactopyranose (34) was obtained using Method B from 7 and 12 in $99 \%$ yield $(\alpha / \beta=$ 11/1). Selected analytical data for $\alpha-34: R_{\mathrm{f}}=0.35$ (ethyl acetate - hexane, $1 / 1, \mathrm{v} / \mathrm{v}$ ); ${ }^{1} \mathrm{H}$-n.m.r.: $\delta, 1.26,1.27,1.30,1.34\left(4 \mathrm{~s}, 12 \mathrm{H}, 4 \times \mathrm{CCH}_{3}\right), 2.01,2.02,2.08\left(3 \mathrm{~s}, 9 \mathrm{H}, 3 \times \mathrm{COCH}_{3}\right), 3.48$ (dd, 1H, J $\left.\mathrm{J}^{\prime}, 3^{\prime}=9.3 \mathrm{~Hz}, \mathrm{H}-2^{\prime}\right), 3.6(\mathrm{~m}, 2 \mathrm{H}, \mathrm{H}-6 \mathrm{a}, 6 \mathrm{~b}), 3.96\left(\mathrm{dd}, 1 \mathrm{H}, \mathrm{J}_{6 \mathrm{a}^{\prime}, 6 \mathrm{~b}^{\prime}}=12.4 \mathrm{~Hz}, \mathrm{H}-6 \mathrm{~b}^{\prime}\right)$, 3.98 (m, 1H, H-5), 4.02 (m, 1H, H-5'), 4.23 (dd, 1H, J $\left.5^{\prime}, 6 \mathrm{a}^{\prime}=4.1 \mathrm{~Hz}, \mathrm{H}-6 \mathrm{a}^{\prime}\right), 4.24$ (dd, 1H, $\left.\mathrm{J}_{2^{\prime}, 3^{\prime}}=9.4 \mathrm{~Hz}, \mathrm{H}-2^{\prime}\right), 4.27\left(\mathrm{dd}, 1 \mathrm{H}, \mathrm{J}_{4,5}=1.9 \mathrm{~Hz}, \mathrm{H}-4\right), 4.51\left(\mathrm{dd}, 1 \mathrm{H}, \mathrm{J}_{3,4}=2.5 \mathrm{~Hz}, \mathrm{H}-3\right), 4.56$ $\left(\mathrm{dd}, 2 \mathrm{H}, \mathrm{J}^{2}=12.2 \mathrm{~Hz}, \mathrm{CH}_{2} \mathrm{Ph}\right), 4.88\left(\mathrm{~d}, 1 \mathrm{H}, \mathrm{J}_{1^{\prime}, 2^{\prime}}=3.6 \mathrm{~Hz}, \mathrm{H}-1^{\prime}\right), 4.90\left(\mathrm{dd}, 1 \mathrm{H}, \mathrm{J}_{4^{\prime}, 5^{\prime}}=9.9 \mathrm{~Hz}\right.$, H-4'), $5.36\left(\mathrm{dd}, 1 \mathrm{H}, \mathrm{J}_{3^{\prime}, 4^{\prime}}=9.6 \mathrm{~Hz}, \mathrm{H}-3^{\prime}\right), 5.43\left(\mathrm{~d}, 1 \mathrm{H}, \mathrm{J}_{1,2}=4.5 \mathrm{~Hz}, \mathrm{H}-1\right) ;{ }^{13} \mathrm{C}-$ n.m.r.: $\delta, 20.9$, $21.0(\times 2), 24.8,25.2,26.3,26.4,62.2,66.5,67.4,67.7,68.8,70.8(\times 2), 71.0,72.2,72.5,96.5$, 97.44, 109.0, 109.4, $127.9(\times 2), 128.2,128.7(\times 2), 138.0,170.1,170.4,171.0$ ppm; HR-FAB MS $[\mathrm{M}+\mathrm{Na}]^{+}$calcd for $\mathrm{C}_{31} \mathrm{H}_{42} \mathrm{NaO}_{14} 661.2472$, found 661.2468 .

Methyl 6-O-(3,4,6-tri- $O$-acetyl-2- $O$-benzyl- $\alpha$-D-glucopyranosyl)-2,3,4-tri- $O$-benzoyl- $\beta$ D-galactopyranoside (35) was obtained using Method B from $\mathbf{7}$ and $\mathbf{1 0}$ in $97 \%$ yield ( $\alpha$ only). Analytical data for $\alpha-\mathbf{3 5}$ were essentially the same as reported previously. ${ }^{9}$

2-Trimethylsilylethyl 4-O-(3,4,6-tri- $O$-acetyl-2- $O$-benzyl- $\alpha$-D-glucopyranosyl)-2,3,6-tri$\boldsymbol{O}$-benzyl- $\boldsymbol{\beta}$-D-galactopyranose (37) was obtained using Method A from $\mathbf{7}$ and $\mathbf{3 6} \mathbf{5}^{55}$ in $88 \%$ yield ( $\alpha$ only). Selected analytical data for $\alpha-37: \mathrm{R}_{\mathrm{f}}=0.39$ (ethyl acetate - hexane, $3 / 7, \mathrm{v} /$ v); ${ }^{1} \mathrm{H}$-n.m.r.: $\delta, 0.03$ (s, 9H, $\left.\mathrm{Si}\left(\mathrm{CH}_{3}\right)_{3}\right), 0.96$ (m, 2H, $\left.\mathrm{CH}_{2} \mathrm{TMS}\right), 1.96,2.01,2.02$ (3 s, 9H, 3 $\left.\times \mathrm{COCH}_{3}\right), 3.41\left(\mathrm{dd}, 1 \mathrm{H}, \mathrm{J}_{3,4}=3.2 \mathrm{~Hz}, \mathrm{H}-3\right), 3.50-3.55\left(\mathrm{~m}, 3 \mathrm{H}, \mathrm{H}-5,-\mathrm{OCH}_{2}{ }^{\mathrm{a}}, \mathrm{H}-6 \mathrm{~b}^{\prime}\right), 3.60$ (dd, $\left.1 \mathrm{H}, \mathrm{J}_{2^{\prime}, 3^{\prime}}=9.9 \mathrm{~Hz}, \mathrm{H}-2^{\prime}\right), 3.70\left(\mathrm{dd}, 1 \mathrm{H}, \mathrm{J}_{2,3}=10.2 \mathrm{~Hz}, \mathrm{H}-2\right), 3.87\left(\mathrm{dd}, 1 \mathrm{H}, \mathrm{J}_{6 \mathrm{a}, 6 \mathrm{~b}}=12.8\right.$ $\mathrm{Hz}, \mathrm{H}-6 \mathrm{a}), 3.99$ (m, 1H, - $\mathrm{OCH}_{2}{ }^{\mathrm{b}}$ ), 4.00 (m, 1H, H-6a'), 4.02 (dd, 1H, H-4), 4.32 (s, 2H, $\left.\mathrm{CH}_{2} \mathrm{Ph}\right), 4.34$ (d, 1H, J $\left.\mathrm{J}_{1,2}=11.3 \mathrm{~Hz}, \mathrm{H}-1\right), 4.40\left(\mathrm{~m}, 1 \mathrm{H}, \mathrm{H}-5^{\prime}\right), 4.61\left(\mathrm{~s}, 2 \mathrm{H}, \mathrm{CH}_{2} \mathrm{Ph}\right), 4.71(\mathrm{~s}$, $\left.2 \mathrm{H}, \mathrm{CH}_{2} \mathrm{Ph}\right), 4.91\left(\mathrm{dd}, 2 \mathrm{H}, \mathrm{J}^{2}=11.1 \mathrm{~Hz}, \mathrm{CH}_{2} \mathrm{Ph}\right), 4.97\left(\mathrm{dd}, 1 \mathrm{H}, \mathrm{J}_{4^{\prime}, 5^{\prime}}=10.2 \mathrm{~Hz}, \mathrm{H}-4^{\prime}\right), 5.11$ $\left(\mathrm{d}, 1 \mathrm{H}, \mathrm{J}_{1^{\prime}, 2^{\prime}}=3.6 \mathrm{~Hz}, \mathrm{H}-1^{\prime}\right), 5.51\left(\mathrm{dd}, 1 \mathrm{H}, \mathrm{J}_{3^{\prime}, 4^{\prime}}=9.6 \mathrm{~Hz}, \mathrm{H}-3^{\prime}\right), 7.19-7.42(\mathrm{~m}, 20 \mathrm{H}$, aromatic) ppm; ${ }^{13}$ C-n.m.r.: $\delta,-1.0(\times 3), 19.0,21.1(\times 2), 21.3,61.8,67.9(\times 2), 68.9,69.0,72.7,73.6$ ( $\times 3), 75.3,76.5,79.5,80.7,99.5,104.1,128.03(\times 6), 128.1,128.2(\times 3), 128.6(\times 2), 128.7$ $(\times 2), 128.8(\times 6), 138.3,138.5,138.6,139.1,170.4(\times 2), 171.0$ ppm; HR-FAB MS [M $+\mathrm{Na}]^{+}$calcd for $\mathrm{C}_{51} \mathrm{H}_{64} \mathrm{NaO}_{14} \mathrm{Si}$ calculated 951.3963 found 951.3961 . 
2-Trimethylsilylethyl 2- $O$-(3,4,6-tri- $O$-acetyl-2-O-benzyl-D-glucopyranosyl)-3-O-

benzyl-4,6- $O$-benzylidene- $\beta$-D-galactopyranoside (38) was obtained using Method A from $\mathbf{7}$ and $\mathbf{1 6}$ colorless syrup in $78 \%$ yield $(\alpha / \beta=3 / 1)$. Selected analytical data for $\alpha-38$ : $R_{f}=0.51$ (ethyl acetate - hexane, $1 / 1$, v/v); ${ }^{1} \mathrm{H}$-n.m.r.: $\delta,-0.40\left(\mathrm{~s}, 9 \mathrm{H}, \mathrm{Si}\left(\mathrm{CH}_{3}\right)_{3}\right), 0.99(\mathrm{~m}, 2 \mathrm{H}$, $\mathrm{CH}_{2}$ TMS), 1.86, 1.93, $1.99\left(3 \mathrm{~s}, 9 \mathrm{H}, 3 \times \mathrm{COCH}_{3}\right), 3.38,\left(\mathrm{~m}, 1 \mathrm{H}, \mathrm{J}_{5}, 6 \mathrm{a}=2.5 \mathrm{~Hz}, \mathrm{~J}_{5}, 6 \mathrm{~b}=4.5\right.$ $\mathrm{Hz}, \mathrm{H}-5), 3.50\left(\mathrm{~m}, 1 \mathrm{H},-\mathrm{OCH}_{2}{ }^{\mathrm{a}}\right), 3.59\left(\mathrm{dd}, 1 \mathrm{H}, \mathrm{J}_{2^{\prime}, 3^{\prime}}=9.6 \mathrm{~Hz}, \mathrm{H}-2^{\prime}\right), 3.70\left(\mathrm{dd}, 1 \mathrm{H}, \mathrm{J}_{3,4}=3.8\right.$ $\mathrm{Hz}, \mathrm{H}-3), 3.77$ (dd, 1H, J $\left.\mathrm{Ja}_{6 \mathrm{~b}}=12.8 \mathrm{~Hz}, \mathrm{H}-6 \mathrm{~b}\right), 3.87$ (dd, 1H, H-6a), $4.00\left(\mathrm{~m}, 1 \mathrm{H},-\mathrm{OCH}_{2}{ }^{\mathrm{b}}\right)$, 4.05 (m, 2H, H-6a', 6b'), 4.10 (dd, 1H, J $2,3=9.4 \mathrm{~Hz}, \mathrm{H}-2$ ), 4.28 (dd, 1H, H-4), 4.38 (d, 1H, $\left.\mathrm{J}_{1,2}=11.3 \mathrm{~Hz}, \mathrm{H}-1\right), 4.58\left(\mathrm{~m}, 1 \mathrm{H}, \mathrm{H}-5^{\prime}\right), 4.89\left(\mathrm{dd}, 1 \mathrm{H}, \mathrm{J}_{4^{\prime}, 5^{\prime}}=10.4 \mathrm{~Hz}, \mathrm{H}-4^{\prime}\right), 5.49(\mathrm{dd}, 1 \mathrm{H}$, $\left.\mathrm{J}_{3^{\prime}, 4^{\prime}}=9.8, \mathrm{H}-3^{\prime}\right), 5.78\left(\mathrm{~d}, 1 \mathrm{H}, \mathrm{J}_{1^{\prime}, 2^{\prime}}=3.6 \mathrm{~Hz}, \mathrm{H}-1^{\prime}\right), 7.26-7.56\left(\mathrm{~m}, 15 \mathrm{H}\right.$, aromatic) ppm; ${ }^{13} \mathrm{C}-$ n.m.r.: $\delta,-1.3,18.8,20.8,21.1,29.9,62.0,66.5,66.8,67.1,68.8,69.5,70.8,71.8,71.9,72.6$, $72.7,95.1,101.4,102.9,126.6(\times 2), 127.7(\times 2), 128.1,128.2,128.3(\times 2), 128.5(\times 2), 128.7$ $(\times 4), 129.2,137.9(\times 2), 138.1,170.0,170.1,171.1 \mathrm{ppm}$; HR-FAB MS $[\mathrm{M}+\mathrm{Na}]^{+}$calcd for $\mathrm{C}_{44} \mathrm{H}_{56} \mathrm{NaO}_{14} \mathrm{Si}$ calculated 859.3337, found 859.3351.

Methyl 6- $O$-(3,4,6-tri- $O$-acetyl-2- $O$-benzyl-D-galactopyranosyl)-2,3,4-tri- $O$-benzoyl- $\beta$ D-galactopyranoside (39) was obtained using Method B from 8 and 10 in $97 \%$ yield $(\alpha / \beta=$ 4/1). Analytical data for $\alpha-39: \mathrm{R}_{\mathrm{f}}=0.54$ (ethyl acetate - hexanes, $1 / 1$, v/v); ${ }^{1} \mathrm{H}-\mathrm{n} . \mathrm{m} . \mathrm{r} .: \mathrm{\delta}, 1.96$, $1.99,2.10\left(3 \mathrm{~s}, 9 \mathrm{H}, 3 \times \mathrm{COCH}_{3}\right), 3.59\left(\mathrm{~s}, 3 \mathrm{H}, \mathrm{OCH}_{3}\right), 3.54(\mathrm{dd}, 1 \mathrm{H}, \mathrm{H}-6 \mathrm{~b}), 3.75(\mathrm{dd}, 1 \mathrm{H}$, $\left.\mathrm{J}_{2^{\prime}, 3^{\prime}}=7.6 \mathrm{~Hz}, \mathrm{H}-2^{\prime}\right), 3.86$ (dd, 1H, $\left.\mathrm{J}_{6 \mathrm{a}, 6 \mathrm{~b}}=10.4 \mathrm{~Hz}, \mathrm{H}-6 \mathrm{a}\right), 4.33\left(\mathrm{dd}, 1 \mathrm{H}, \mathrm{H}-6 \mathrm{~b}^{\prime}\right), 4.67$ (dd, $1 \mathrm{H}$, $\left.\mathrm{J}_{6 \mathrm{a}^{\prime}, 6 \mathrm{~b}^{\prime}}=10.7 \mathrm{~Hz}, \mathrm{H}-6 \mathrm{a}^{\prime}\right), 4.21\left(\mathrm{~m}, 1 \mathrm{H}, \mathrm{J}_{5,6 \mathrm{a}}=4.3 \mathrm{~Hz}, \mathrm{~J}_{5,6 \mathrm{~b}}=2.8 \mathrm{~Hz}, \mathrm{H}-5\right), 4.31(\mathrm{dd}, 1 \mathrm{H}$, $\left.\mathrm{J}_{5^{\prime}, 6 \mathrm{a}^{\prime}}=4.2 \mathrm{~Hz}, \mathrm{~J}_{5^{\prime}, 6 \mathrm{~b}^{\prime}}=3.1 \mathrm{~Hz}, \mathrm{H}-5^{\prime}\right), 4.65\left(\mathrm{~s}, 2 \mathrm{H}, \mathrm{CH}_{2} \mathrm{Ph}\right), 4.69\left(\mathrm{~d}, 1 \mathrm{H}, \mathrm{J}_{1,2}=8.1 \mathrm{~Hz}, \mathrm{H}-1\right)$, $4.76\left(\mathrm{~d}, 1 \mathrm{H}, \mathrm{J}_{1^{\prime}, 2^{\prime}}=3.4, \mathrm{H}-1^{\prime}\right), 5.25\left(\mathrm{dd}, 1 \mathrm{H}, \mathrm{J}_{3^{\prime}, 4^{\prime}}=3.8 \mathrm{~Hz}, \mathrm{H}-3^{\prime}\right), 5.36\left(\mathrm{dd}, 1 \mathrm{H}, \mathrm{J}_{4^{\prime}, 5^{\prime}}=0.8 \mathrm{~Hz}\right.$, H-4'), 5.49 (dd, $\left.1 \mathrm{H}, \mathrm{J}_{3,4}=3.2 \mathrm{~Hz}, \mathrm{H}-3\right), 5.69$ (dd, $\left.1 \mathrm{H}, \mathrm{J}_{2,3}=9.9 \mathrm{~Hz}, \mathrm{H}-2\right), 5.80$ (dd, $1 \mathrm{H}, \mathrm{J}_{4,5}=$ $1.1 \mathrm{~Hz}, \mathrm{H}-4), 7.21-8.09$ (m, 20H, aromatic) ppm; ${ }^{13}$ C-n.m.r.: $\delta, 20.8,20.9,21.0,29.9,57.5$, 66.9, 67.0, 68.7, 69.1, 69.7, 70.1, 72.0, 72.9, 73.4, 73.6, 97.6, 102.6, $128.2(\times 2), 128.5(\times 2)$, $128.6(\times 2), 128.7(\times 2), 128.9(\times 2), 129.1,129.3,129.6,130.0(\times 4), 130.2(\times 2), 133.4(\times$ 2), 133.9, 138.2, 142.1, 142.4, 142.6, 165.7 (× 2), 165.9, 170.2, 170.3, 170.6 ppm; HR-FAB MS $[\mathrm{M}+\mathrm{Na}]^{+}$calcd for $\mathrm{C}_{47} \mathrm{H}_{48} \mathrm{NaO}_{17}$ 907.2789, found 907.2798.

6- $O$-(3,4,6-Tri- $O$-acetyl-2- $O$-benzyl-D-galactopyranosyl)-1,2:3,4-di- $O$-isopropylidene- $\alpha$ D-galactopyranose (40) was obtained using Method B from 8 and 12 in $99 \%$ yield $(\alpha / \beta 5 / 1)$. Selected analytical data for $\alpha-\mathbf{4 0}: \mathrm{R}_{\mathrm{f}}=0.35$ (ethyl acetate - hexane, $1 / 1$, v/v); ${ }^{1} \mathrm{H}$-n.m.r: $\delta, 1.29$, $1.33,1.45,1.56\left(4 \mathrm{~s}, 12 \mathrm{H}, 4 \times \mathrm{CCH}_{3}\right), 1.99,2.05,2.11\left(3 \mathrm{~s}, 9 \mathrm{H}, 3 \times \mathrm{COCH}_{3}\right), 3.48(\mathrm{dd}, 1 \mathrm{H}$, $\left.\mathrm{J}_{2^{\prime}, 3^{\prime}}=9.3 \mathrm{~Hz}, \mathrm{H}-2^{\prime}\right), 3.6(\mathrm{~m}, 2 \mathrm{H}, \mathrm{H}-6 \mathrm{a}, 6 \mathrm{~b}), 3.96\left(\mathrm{dd}, 1 \mathrm{H}, \mathrm{J}_{6 \mathrm{a}^{\prime}, 6 \mathrm{~b}^{\prime}}=12.4 \mathrm{~Hz}, \mathrm{H}-6 \mathrm{~b}^{\prime}\right), 3.98(\mathrm{~m}$, $1 \mathrm{H}, \mathrm{H}-5), 4.02\left(\mathrm{~m}, 1 \mathrm{H}, \mathrm{H}-5^{\prime}\right), 4.23\left(\mathrm{dd}, 1 \mathrm{H}, \mathrm{J}_{5^{\prime}, 6 \mathrm{a}^{\prime}}=4.1 \mathrm{~Hz}, \mathrm{H}-6 \mathrm{a}^{\prime}\right), 4.24\left(\mathrm{dd}, 1 \mathrm{H}, \mathrm{J}_{2^{\prime}, 3^{\prime}}=9.4\right.$ $\left.\mathrm{Hz}, \mathrm{H}-2^{\prime}\right), 4.27$ (dd, 1H, J $\left.{ }_{4,5}=1.9 \mathrm{~Hz}, \mathrm{H}-4\right), 4.51$ (dd, 1H, J $\left.{ }_{3,4}=2.5 \mathrm{~Hz}, \mathrm{H}-3\right), 4.56$ (dd, 2H, J $\left.=12.2 \mathrm{~Hz}, \mathrm{CH}_{2} \mathrm{Ph}\right), 4.88\left(\mathrm{~d}, 1 \mathrm{H}, \mathrm{J}_{1^{\prime}, 2^{\prime}}=3.6 \mathrm{~Hz}, \mathrm{H}-1^{\prime}\right), 4.90\left(\mathrm{dd}, 1 \mathrm{H}, \mathrm{J}_{4^{\prime}, 5^{\prime}}=9.9 \mathrm{~Hz}, \mathrm{H}-4^{\prime}\right), 5.36$ $\left(\mathrm{dd}, 1 \mathrm{H}, \mathrm{J}_{3^{\prime}, 4^{\prime}}=9.6 \mathrm{~Hz}, \mathrm{H}-3^{\prime}\right), 5.43\left(\mathrm{~d}, 1 \mathrm{H}, \mathrm{J}_{1,2}=4.5 \mathrm{~Hz}, \mathrm{H}-1\right)$ ppm; ${ }^{13} \mathrm{C}-$-n.m.r.: $\delta, 20.9,21.0$, 21.1, 24.8, 25.2, 26.3, 26.4, 62.2, 66.5, 67.4, 67.7, 68.8, $70.8(\times 2), 71.0,72.2,72.5,96.5,97.4$, 109.0, 109.4, $127.9(\times 2), 128.2,128.7(\times 2), 138.0,170.1,170.4,171.0$ ppm; HR-FAB MS $[\mathrm{M}+\mathrm{Na}]^{+}$calcd for $\mathrm{C}_{31} \mathrm{H}_{42} \mathrm{NaO}_{14}$ 661.2472, found 661.2468.

Benzoxazolyl 2,3,4-tri- $O$-benzoyl-6- $O$-(2,3,4,6-tetra- $O$-benzyl-D-glucopyranosyl)-1-thio$\boldsymbol{\beta}$-D-glucopyranoside (47) was obtained using Method $C$ from 5 and $\mathbf{4 6}$ as a colorless syrup in $64 \%$ yield $(\alpha / \beta=3 / 1)$. Selected analytical data for $\alpha-47$ : $R_{\mathrm{f}}=0.40$ (ethyl acetate - toluene, 1/9, v/v); ${ }^{1} \mathrm{H}-$ n.m.r.: $\delta, 3.42-3.52(\mathrm{~m}, 4 \mathrm{H}), 3.66$ (dd, $\left.1 \mathrm{H}, \mathrm{H}-6 \mathrm{~b}^{\prime}\right), 3.74-3.83(\mathrm{~m}, 2 \mathrm{H}), 3.89$ (dd, $\left.1 \mathrm{H}, \mathrm{J}_{6 \mathrm{a}^{\prime}, 6 \mathrm{~b}^{\prime}}=9.2 \mathrm{~Hz}, \mathrm{H}-6 \mathrm{a}^{\prime}\right), 4.23-4.34(\mathrm{~m}, 2 \mathrm{H}), 4.39\left(\mathrm{~m}, 1 \mathrm{H}, \mathrm{J}_{5^{\prime}, 6 \mathrm{a}^{\prime}}=6.8 \mathrm{~Hz}, \mathrm{~J}_{5^{\prime}, 6 \mathrm{~b}^{\prime}}=2.3 \mathrm{~Hz}\right.$, H-5'), 4.43-4.47 (m, 2H), 4.57 (dd, 2H, J $\left.{ }^{2}=12.1 \mathrm{~Hz}, \mathrm{CH}_{2} \mathrm{Ph}\right), 4.70\left(\mathrm{~d}, 1 \mathrm{H}, \mathrm{J}_{1,2}=1.6 \mathrm{~Hz}, \mathrm{H}-1\right)$, 4.73-4.77 (m, 2H), $5.64\left(\mathrm{dd}, 1 \mathrm{H}, \mathrm{J}_{4^{\prime}, 5^{\prime}}=10.0 \mathrm{~Hz}, \mathrm{H}-4^{\prime}\right), 5.70\left(\mathrm{dd}, 1 \mathrm{H}, \mathrm{J}_{3^{\prime}, 4^{\prime}}=10.0 \mathrm{~Hz}, \mathrm{H}-3^{\prime}\right)$, $6.03\left(\mathrm{dd}, 1 \mathrm{H}, \mathrm{J}_{2^{\prime}, 3^{\prime}}=9.4 \mathrm{~Hz}, \mathrm{H}-2^{\prime}\right), 6.07\left(\mathrm{~d}, 1 \mathrm{H}, \mathrm{J}_{1^{\prime}, 2^{\prime}}=10.6 \mathrm{~Hz}, \mathrm{H}-1^{\prime}\right), 7.01-7.98(\mathrm{~m}, 39 \mathrm{H}$, aromatic) ppm; ${ }^{13}$ C-n.m.r.: $\delta, 66.5,68.5,69.6,70.2,70.7,73.4,73.4,74.3,74.7,75.7,78.0$, $80.1,82.1,84.0,97.1,110.4,119.0,124.4,124.6,127.5,127.6,127.7,127.9(\times 4), 128.0(\times$ 
2), $128.1(\times 3), 128.3(\times 2), 128.3(\times 3), 128.5(\times 4), 128.5(\times 2), 128.6(\times 2), 128.6(\times 2), 128.7$ $(\times 2), 128.8,128.9,129.1,130.1(\times 5), 133.5,133.7,138.2,138.5,138.9,139.2,141.8,152.2$, 161.4, 165.2, 165.5, 165.9 ppm; HR-FAB MS [M+Na $]^{+}$calcd for $\mathrm{C}_{68} \mathrm{H}_{61} \mathrm{NNaO}_{14} \mathrm{~S} 1170.3710$, found 1170.3724 .

Ethyl 6-O-(3,4,6-tri- $O$-acetyl-2- $O$-benzyl- $\alpha$-D-glucopyranosyl)-2,3,4-tri- $O$-benzoyl-1thio- $\beta$-D-galactopyranoside (50) was obtained from 7 and 4956 using Method B in $98 \%(\alpha$ only) as a colorless syrup. Analytical data for 50: $\mathrm{R}_{\mathrm{f}}=0.51$ (ethyl acetate - hexane, 1/1, v/v); $[\alpha]_{\mathrm{D}}{ }^{22} 140.0^{\circ}\left(\mathrm{c}=1.0, \mathrm{CHCl}_{3}\right) ;{ }^{1} \mathrm{H}$-n.m.r: $\delta, 1.35(\mathrm{dd}, 3 \mathrm{H}), 2.01,2.01,2.18(3 \mathrm{~s}, 9 \mathrm{H}, 3 \times$ $\left.\mathrm{COCH}_{3}\right), 2.84(\mathrm{~m}, 2 \mathrm{H}), 3.54-3.65(\mathrm{~m}, 2 \mathrm{H}), 3.74(\mathrm{~s}, 2 \mathrm{H}), 3.86(\mathrm{dd}, 1 \mathrm{H}, \mathrm{J}=7.2, \mathrm{~J}=10.4 \mathrm{~Hz})$, $4.04(\mathrm{dd}, 1 \mathrm{H}, \mathrm{J}=2.0, \mathrm{~J}=12.4 \mathrm{~Hz}), 4.17(\mathrm{~m}, 1 \mathrm{H}), 4.24-4.30(\mathrm{~m}, 2 \mathrm{H}), 4.62(\mathrm{dd}, 2 \mathrm{H}, \mathrm{J}=12.5$ $\mathrm{Hz}), 4.77(\mathrm{~d}, 1 \mathrm{H}, \mathrm{J}=3.5 \mathrm{~Hz}), 4.85(\mathrm{~d}, 1 \mathrm{H}, \mathrm{J}=10.0 \mathrm{~Hz}), 4.97(\mathrm{dd}, 1 \mathrm{H}, \mathrm{J}=10.4 \mathrm{~Hz}), 5.44$ (dd, $1 \mathrm{H}, \mathrm{J}=9.6 \mathrm{~Hz}), 5.60(\mathrm{dd}, 1 \mathrm{H}, \mathrm{J}=3.3, \mathrm{~J}=10.0 \mathrm{~Hz}), 5.81(\mathrm{dd}, 1 \mathrm{H}, \mathrm{J}=5.0 \mathrm{~Hz}), 5.90(\mathrm{~d}, 1 \mathrm{H}, \mathrm{J}$ $=3.3 \mathrm{~Hz}), 7.20-8.10(\mathrm{~m}, 20 \mathrm{H})$ ppm; ${ }^{13} \mathrm{C}-$ n.m.r: $\delta, 20.8(\times 2), 21.0,24.9,29.5,29.6,29.9,31.9$, $32.1,54.0,62.0,67.3,67.6,68.5,68.7,69.3,69.7,72.0,73.0,73.3,76.6,76.7,84.4,96.9,128.2$, $128.3,128.5,128.6,128.7,128.9,129.0,129.4,129.9,130.1,133.4,133.5,133.9,137.8,165.6$ $(\times 2), 165.8,169.9,170.2,170.8$ ppm; HR-FAB MS $[\mathrm{M}+\mathrm{Na}]^{+}$calcd for $\mathrm{C}_{48} \mathrm{H}_{50} \mathrm{NaO}_{16} \mathrm{~S}$ 937.2717, found 937.2717 .

Pent-4-enyl $O$-(3,4,6-tri- $O$-acetyl-2- $O$-benzyl- $\alpha$-D-glucopyranosyl)-(1 $\rightarrow 6)-O$ - $(2,3,4$-tri$O$-benzoyl- $\beta$-D-galactopyranosyl)-( $\rightarrow 6)$-2,3,4-tri- $O$-benzyl- $\beta$-D-glucopyranoside $(51)$ was prepared from $\mathbf{4 8}$ and $\mathbf{5 0}$ using Method A in $92 \%$ yield as a colorless syrup. Analytical data for 51: $\mathrm{R}_{\mathrm{f}}=0.57$ (ethyl acetate - hexane, 1/1, v/v); $[\alpha]_{\mathrm{D}}{ }^{22} 98.9^{\circ}\left(\mathrm{c}=1.0, \mathrm{CHCl}_{3}\right) ;{ }^{1} \mathrm{H}$ n.m.r: $\delta, 1.61-1.74(\mathrm{~m}, 2 \mathrm{H}), 1.94,1.97,1.98\left(3 \mathrm{~s}, 9 \mathrm{H}, 3 \times \mathrm{COCH}_{3}\right), 2.05-2.18(\mathrm{~m}, 2 \mathrm{H}), 3.31-$ $3.46(\mathrm{~m}, 4 \mathrm{H}), 3.51-3.56(\mathrm{~m}, 2 \mathrm{H}), 3.63(\mathrm{dd}, 1 \mathrm{H}, \mathrm{J}=6.0, \mathrm{~J}=10.5 \mathrm{~Hz}), 3.80-3.86(\mathrm{~m}, 3 \mathrm{H}), 3.97-$ $4.12(\mathrm{~m}, 3 \mathrm{H}), 4.22-4.29(\mathrm{~m}, 3 \mathrm{H}), 4.44-4.67(\mathrm{~m}, 6 \mathrm{H}), 4.78-5.03(\mathrm{~m}, 7 \mathrm{H}), 5.42$ (dd, 1H, J = 9.6 $\mathrm{Hz}), 5.53$ (dd, 1H, J = 3.5, J = 10.4 Hz), 5.76-5.84 (m, 2H), 5.87 (dd, 1H, J = 3.2 Hz), 7.108.15 (m, 35H) ppm; ${ }^{13}$ C-n.m.r: $\delta, 20.8,21.0,29.1,29.9,30.4,32.1,62.0,66.6,67.8,68.4,68.7$, 68.8, 69.4, 70.2, 72.0, 72.6, 73.1, 74.9, 75.1, 75.7, 76.6, 77.8, 82.3, 84.7, 97.2, 101.8, 103.7, $115.2,127.7,127.9,128.0,128.3,128.5,128.6,128.7,128.9,129.2,129.9,129.9,130.3,133.4$, $133.8,137.9,138.2,138.6,138.8,165.4,165.6,165.9,169.9,170.7(\times 2)$ ppm; HR-FAB MS $[\mathrm{M}+\mathrm{Na}]^{+}$calcd for $\mathrm{C}_{78} \mathrm{H}_{82} \mathrm{NaO}_{22} 1393.5196$, found 1393.5216 .

Ethyl 2,3,4-tri- $O$-benzoyl-6- $O$-(2,3,4,6-tetra- $O$-benzoyl- $\beta$-D-glucopyranosyl)-1-thio- $\beta$-Dgalactopyranoside (52) was obtained using Method A from 2 and $\mathbf{4 9}^{56}$ in $99 \%$ yield. Analytical data for $\mathbf{5 2}$ were essentially the same as reported previously. ${ }^{13}$

Pent-4-enyl $O$-(2,3,4,6-tetra- $O$-benzoyl- $\beta$-D-glucopyranosyl)-(1 $\rightarrow 6)-O$ - $(2,3,4-$ tri- $O$ benzoyl- $\beta$-D-galactopyranosyl)- $(1 \rightarrow 6)-3-O$-benzoyl-2-deoxy-2-phthalimido- $\beta$-Dglucopyranoside (54) was prepared from 50 and 51 using Method A in $90 \%$ yield as a colorless syrup. Analytical data for 54: $\mathrm{R}_{\mathrm{f}}=0.47$ (ethyl acetate - hexane, $\left.1 / 1, \mathrm{v} / \mathrm{v}\right) ;[\alpha]_{\mathrm{D}} 2290.1^{\circ}(\mathrm{c}=$ 0.6, $\left.\mathrm{CHCl}_{3}\right)$; ${ }^{1} \mathrm{H}$-n.m.r: $\delta, 1.27\left(\mathrm{~m}, 2 \mathrm{H},-\mathrm{OCH}_{2} \mathrm{CH}_{2} \mathrm{CH}_{2}\right), 1.67\left(\mathrm{~m}, 2 \mathrm{H}-\mathrm{OCH}_{2} \mathrm{CH}_{2} \mathrm{CH}_{2}\right), 3.18$ $\left(\mathrm{m}, 1 \mathrm{H}, \mathrm{OCH}_{2}{ }^{\mathrm{b}}\right), 3.50\left(\mathrm{~m}, 1 \mathrm{H}, \mathrm{OCH}_{2}{ }^{\mathrm{a}}\right), 3.60(\mathrm{~m}, 1 \mathrm{H}, \mathrm{H}-6 \mathrm{~b}), 3.68(\mathrm{~m}, 1 \mathrm{H}, \mathrm{H}-4), 3.71(\mathrm{~m}, 1 \mathrm{H}$, H-6a), 3.90 (m, 1H, H-5'), 3.94 (m, 1H, H-6b'), 4.06 (m, 1H, H-6a'), 4.09 (m, 1H, J J",6a" = 3.2

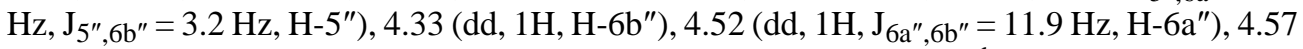
$\left(\mathrm{m}, 1 \mathrm{H},=\mathrm{CH}_{2}^{\mathrm{a}}\right), 4.63\left(\mathrm{~d}, 1 \mathrm{H}, \mathrm{J}_{1^{\prime}, 2^{\prime}}=7.9 \mathrm{~Hz}, \mathrm{H}-1^{\prime}\right), 4.66\left(\mathrm{~m}, 1 \mathrm{H},=\mathrm{CH}_{2}{ }^{\mathrm{b}}\right), 5.08\left(\mathrm{~d}, 1 \mathrm{H}, \mathrm{J}_{1^{\prime \prime}, 2^{\prime \prime}}=\right.$ $\left.8.1 \mathrm{~Hz}, \mathrm{H}-1^{\prime \prime}\right), 5.23\left(\mathrm{~d}, 1 \mathrm{H}, \mathrm{J}_{1,2}=7.7 \mathrm{~Hz}, \mathrm{H}-1\right), 5.41\left(\mathrm{dd}, 1 \mathrm{H}, \mathrm{J}_{3^{\prime}, 4^{\prime}}=9.8 \mathrm{~Hz}, \mathrm{H}-3^{\prime}\right), 5.44(\mathrm{~m}$, $1 \mathrm{H},-\mathrm{CH}=), 5.46\left(\mathrm{dd}, 1 \mathrm{H}, \mathrm{J}_{2^{\prime \prime}, 3^{\prime \prime}}=9.8 \mathrm{~Hz}, \mathrm{H}-2^{\prime \prime}\right), 5.61\left(\mathrm{dd}, 1 \mathrm{H}, \mathrm{J}_{4^{\prime \prime}, 5^{\prime \prime}}=9.4 \mathrm{~Hz}, \mathrm{H}-4^{\prime \prime}\right), 5.70$ (dd, $\left.1 \mathrm{H}, \mathrm{J}_{2^{\prime}, 3^{\prime}}=8.1 \mathrm{~Hz}, \mathrm{H}-2^{\prime}\right), 5.75\left(\mathrm{~m}, 1 \mathrm{H}, \mathrm{H}-4^{\prime}\right), 5.77\left(\mathrm{dd}, 1 \mathrm{H}, \mathrm{J}_{3,4}=9.8 \mathrm{~Hz}, \mathrm{H}-3\right), 5.81(\mathrm{dd}, 1 \mathrm{H}$, $\left.\mathrm{J}_{3^{\prime \prime}, 4^{\prime \prime}}=9.6 \mathrm{~Hz}, \mathrm{H}-3^{\prime \prime}\right), 7.10-8.00\left(\mathrm{~m}, 59 \mathrm{H}\right.$, aromatic) ppm; ${ }^{13} \mathrm{C}-$ n.m.r.: $\delta, 28.6,29.9,30.0,31.1$, 68.2, 69.0, 69.1, $69.6(\times 2), 69.9,70.8,71.8,71.9,72.5,73.3,74.6,75.0(\times 2), 98.1,101.4$, $101.9,114.8,123.7(\times 2), 128.5(\times 14), 128.8,129.0(\times 6), 129.2,129.4,129.5,129.8(\times 3)$, $130.0(\times 10), 130.1(\times 3), 130.2(\times 3), 133.4(\times 2), 133.5(\times 2), 133.7,133.8,134.3(\times 2), 138.0$ 
(×2), 165.3, 165.3, 165.4, 165.6, 165.8, 166.3, 166.5, 166.9, $207.2(\times 2)$ ppm; HR-FAB MS $[\mathrm{M}+\mathrm{Na}]^{+}$calcd for $\mathrm{C}_{87} \mathrm{H}_{75} \mathrm{NaNO}_{25}$ 1556.4526, found 1556.4497.

Methyl $O$-(2,3,4,6-tetra- $O$-benzoyl- $\beta$-D-glucopyranosyl)-( $1 \rightarrow 6)-O$-(2,3,4-tri- $O$-benzoyl$\beta$-D-galactopyranosyl)-(1 $\rightarrow 6)-O$ - $(3-O$-benzoyl-2-deoxy-2-phthalimido- $\beta$-Dglucopyranosyl)-2,3,4-tri- $\boldsymbol{O}$-benzyl- $\boldsymbol{\alpha}$-D-glucopyranoside (55) was obtained from $\mathbf{5 4}$ and 14 using conventional NIS/TMSOTf activation (see supporting information) in 73\% yield as a colorless syrup. Selected analytical data for $\mathbf{5 5}: \mathrm{R}_{\mathrm{f}}=0.5$ (ethyl acetate - hexane, 1/1, v/v); $[\alpha]_{\mathrm{D}}{ }^{22} 26.1^{\circ}\left(\mathrm{c}=1.0, \mathrm{CHCl}_{3}\right) ;{ }^{1} \mathrm{H}-\mathrm{n} . \mathrm{m} . \mathrm{r} .:$, $, 3.06(\mathrm{~s}, 3 \mathrm{H}), 3.13(\mathrm{~m}, 1 \mathrm{H}), 3.28(\mathrm{dd}, 1 \mathrm{H}, \mathrm{J}=3.4$, $9.6 \mathrm{~Hz}), 3.34(\mathrm{dd}, 1 \mathrm{H}, \mathrm{J}=3.1 \mathrm{~Hz}), 3.42-3.48(\mathrm{~m}, 2 \mathrm{H}), 3.62-3.77(\mathrm{~m}, 4 \mathrm{H}), 3.87-4.24(\mathrm{~m}, 7 \mathrm{H})$, $4.26-4.36(\mathrm{~m}, 3 \mathrm{H}), 4.52(\mathrm{dd}, 1 \mathrm{H}, \mathrm{J}=3.2 \mathrm{~Hz}), 4.56(\mathrm{dd}, 2 \mathrm{H}, \mathrm{J}=12.2 \mathrm{~Hz}), 4.66(\mathrm{~d}, 1 \mathrm{H}, \mathrm{J}=7.9$ $\mathrm{Hz}), 4.67(\mathrm{dd}, 2 \mathrm{H}, \mathrm{J}=10.9 \mathrm{~Hz}), 5.01(\mathrm{~d}, 1 \mathrm{H}, \mathrm{J}=7.8 \mathrm{~Hz}), 5.30(\mathrm{~d}, 1 \mathrm{H}, \mathrm{J}=8.4 \mathrm{~Hz}), 5.40$ (dd, $1 \mathrm{H}, \mathrm{J}=3.5, \mathrm{~J}=10.4 \mathrm{~Hz}), 5.46(\mathrm{dd}, 1 \mathrm{H}, \mathrm{J}=7.9, \mathrm{~J}=9.7 \mathrm{~Hz}), 5.6(\mathrm{dd}, 1 \mathrm{H}, \mathrm{J}=8.4 \mathrm{~Hz}), 5.66(\mathrm{dd}$, $1 \mathrm{H}, \mathrm{J}=7.8, \mathrm{~J}=10.4 \mathrm{~Hz}), 5.72-5.76(\mathrm{~m}, 2 \mathrm{H}), 5.80(\mathrm{dd}, 1 \mathrm{H}, \mathrm{J}=9.5 \mathrm{~Hz}), 6.87-8.01(\mathrm{~m}, 59 \mathrm{H})$ ppm; ${ }^{13}$ C-n.m.r.: $\delta, 29.9,54.6,55.3,62.9,68.1,68.3,69.0,69.3,69.4,69.6,69.6,70.1,70.6$, $71.8,71.9,72.5,73.2,73.6,74.3,74.9,75.3,75.8,80.0,82.0,98.1,98.2,101.4,102.0,127.7-$ 139.0 (72 signals), 165.3, 165.3, 165.4, 165.6, 165.8, 166.3, 166.3, 166.9 ppm; HR-FAB MS $[\mathrm{M}+\mathrm{Na}]^{+}$calcd for $\mathrm{C}_{110} \mathrm{H}_{97} \mathrm{NaNO}_{30}$ 1934.5993, found 1934.5965.

\section{Supplementary Material}

Refer to Web version on PubMed Central for supplementary material.

\section{Acknowledgements}

The authors thank the National Institutes of General Medical Sciences (GM077170) and the University of Missouri St. Louis Graduate School Dissertation Fellowship (to M.N.K.) for financial support of this research; NSF for grants to purchase the NMR spectrometer (CHE-9974801) and the mass spectrometer (CHE-9708640) used in this work; Dr. R. S. Luo for assistance with $500 \mathrm{MHz}$ 2D n.m.r. experiments; and Dr. R. E. K. Winter and Mr. J. Kramer for HRMS determinations.

\section{References}

1. El Ashry ESH, Awad LF, Atta AI. Tetrahedron 2006;62:2943-2998.

2. Mukaiyama T, Nakatsuka T, Shoda SI. Chem Lett 1979:487-490.

3. Hanessian S, Bacquet C, Lehong N. Carbohydr Res 1980;80:c17-c22.

4. Woodward RB, Logusch E, Nambiar KP, Sakan K, Ward DE, Au-Yeung BW, Balaram P, Browne LJ, Card PJ, Chen CH. J Am Chem Soc 1981;103:3215-3217.

5. Reddy GV, Kulkarni VR, Mereyala HB. Tetrahedron Lett 1989;30:4283-4286.

6. Chen Q, Kong F. Carbohydr Res 1995;272:149-157.

7. Tsuboyama K, Takeda K, Torii K, Ebihara M, Shimizu J, Suzuki A, Sato N, Furuhata K, Ogura H. Chem Pharm Bull 1990;38:636-638.

8. Pornsuriyasak P, Kamat MN, Demchenko AV. ACS Symp Ser 2007;960:165-189.

9. Pornsuriyasak P, Demchenko AV. Chem Eur J 2006;12:6630-6646.

10. Demchenko AV, Pornsuriyasak P, De Meo C, Malysheva NN. Angew Chem, Int Ed 2004;43:30693072.

11. Demchenko AV, Malysheva NN, De Meo C. Org Lett 2003;5:455-458. [PubMed: 12583742]

12. Kamat MN, Demchenko AV. Org Lett 2005;7:3215-3218. [PubMed: 16018624]

13. Pornsuriyasak P, Demchenko AV. Tetrahedron: Asymmetry 2005;16:433-439.

14. Smoot JT, Pornsuriyasak P, Demchenko AV. Angew Chem Int Ed 2005:7123-7126.

15. Pornsuriyasak P, Gangadharmath UB, Rath NP, Demchenko AV. Org Lett 2004;6:4515-4518. [PubMed: 15548064] 
16. Ferrieres V, Blanchard S, Fischer D, Plusquellec D. Bioorg Med Chem Lett 2002;12:3515-3518. [PubMed: 12443765]

17. Euzen R, Ferrieres V, Plusquellec D. J Org Chem 2005;70:847-855. [PubMed: 15675842]

18. Ramakrishnan A, Pornsuriyasak P, Demchenko AV. J Carbohydr Chem 2005;24:649-663.

19. Kamat MN, Rath NP, Demchenko AV. J Org Chem. preceding article in this issue, in press

20. Demchenko A, Stauch T, Boons GJ. Synlett 1997:818-820.

21. Demchenko AV. Curr Org Chem 2003;7:35-79.

22. Demchenko AV. Synlett 2003:1225-1240.

23. Nitz, M.; Bundle, DR. Glycoscience: Chemistry and Chemical Biology. Fraser-Reid, B.; Tatsuta, K.; Thiem, J., editors. 2. Springer; Berlin - Heidelberg - New York: 2001. p. 1497-1542.

24. Igarashi K. Adv Carbohydr Chem Biochem 1977;34:243-283.

25. Schmidt, RR.; Jung, KH. Carbohydrates in Chemistry and Biology. Ernst, B.; Hart, GW.; Sinay, P., editors. 1. Wiley-VCH; Weinheim, New York: 2000. p. 5-59.

26. Zhang, Z.; Wong, CH. Carbohydrates in Chemistry and Biology. Ernst, B.; Hart, GW.; Sinay, P., editors. 1. Wiley-VCH; Weinheim, New York: 2000. p. 117-134.

27. Plante OJ, Palmacci ER, Andrade RB, Seeberger PH. J Am Chem Soc 2001;123:9545-9554. [PubMed: 11572674]

28. Nicolaou, KC.; Ueno, H. Preparative Carbohydrate Chemistry. Hanessian, S., editor. Marcel Dekker, Inc.; New York: 1997. p. 313-338.

29. Mukaiyama T. Angew Chem Int Ed 2004;43:5590-5614.

30. Garegg PJ. Adv Carbohydr Chem Biochem 1997;52:179-205. [PubMed: 9218334]

31. Oscarson, S. Carbohydrates in Chemistry and Biology. Ernst, B.; Hart, GW.; Sinay, P., editors. 1. Wiley-VCH; Weinheim, New York: 2000. p. 93-116.

32. Fraser-Reid, B.; Anilkumar, G.; Gilbert, MB.; Joshi, S.; Kraehmer, R. Carbohydrates in Chemistry and Biology. Ernst, B.; Hart, GW.; Sinay, P., editors. 1. Wiley-VCH; Weinheim, New York: 2000. p. 135-154.

33. Boons, GJ. Glycoscience: Chemistry and Chemical Biology. Fraser-Reid, B.; Tatsuta, K.; Thiem, J., editors. 1. Springer; Berlin - Heidelberg - New York: 2001. p. 551-581.

34. Mehta S, Pinto BM. J Org Chem 1993;58:3269-3276.

35. Wolfrom, ML.; Thompson, A. Methods in Carbohydrate Chemistry. Whistler, RL.; Wolfrom, ML., editors. 2. Academic Press; New York - London: 1963. p. 215-220.

36. Greene, TW.; Wuts, PGM. Protective Groups in Organic Synthesis. 3. Wiley; New York: 1999.

37. Demchenko AV. Lett Org Chem 2005;2:580-589.

38. Mootoo DR, Konradsson P, Udodong U, Fraser-Reid B. J Am Chem Soc 1988;110:5583-5584.

39. Fraser-Reid B, Udodong UE, Wu ZF, Ottosson H, Merritt JR, Rao CS, Roberts C, Madsen R. Synlett 1992:927-942. and references therein

40. Demchenko AV, De Meo C. Tetrahedron Lett 2002;43:8819-8822.

41. Ziegler T, Sutoris H, Glaudemans CPJ. Carbohydr Res 1992;229:271-291. [PubMed: 1382854]

42. Veeneman GH, van Boom JH. Tetrahedron Lett 1990;31:275-278.

43. Hashimoto S, Honda T, Ikegami S. J Chem Soc, Chem Commun 1989:685-687.

44. Jansson K, Ahlfors S, Frejd T, Kihlberg J, Magnusson G, Dahmen J, Noori G, Stenvall K. J Org Chem 1988;53:5629-5647.

45. Kondo Y. Agric and Biol Chem 1975;39:1879-1881.

46. Dasgupta F, Garegg PJ. Carbohydr Res 1988;177:C13-C17.

47. Garegg PJ, Hultberg H. Carbohydr Res 1981;93:C10-C11.

48. Codee JDC, Van den Bos LJ, Litjens REJN, Overkleeft HS, Van Boeckel CAA, Van Boom JH, Van der Marel GA. Tetrahedron 2004;60:1057-1064.

49. Eby R, Schuerch C. Carbohydr Res 1975;39:33-38.

50. Grayson EJ, Ward SJ, Hall AL, Rendle PM, Gamblin DP, Batsanov AS, Davis BG. J Org Chem 2005;70:9740-9754. [PubMed: 16292802]

51. Dasgupta F, Garegg PJ. Carbohydr Res 1990;202:225-238. [PubMed: 2171766] 
52. Sollogoub M, Das SK, Mallet JM, Sinay P. C R Acad Sci Ser 2 1999;2:441-448.

53. Pougny JR, Nassr MAM, Naulet N, Sinay P. Nouveau J Chem 1978;2:389-395.

54. Kim KS, Lee YJ, Kim HY, Kang SS, Kwon SY. Org Biomol Chem 2004;2:2408-2410. [PubMed: 15326517]

55. Nilsson U, Ray AK, Magnusson G. Carbohydr Res 1994;252:117-136. [PubMed: 8137356]

56. Birberg W, Lonn H. Tetrahedron Lett 1991;32:7453-7456. 


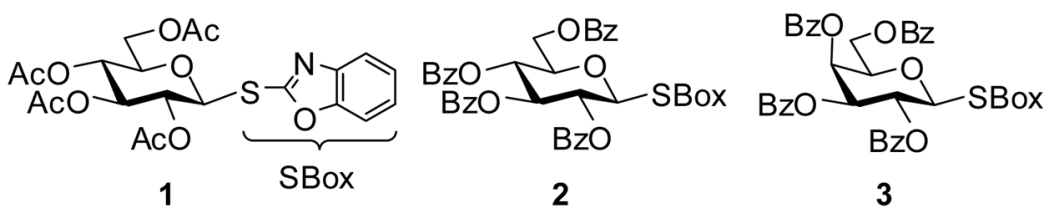

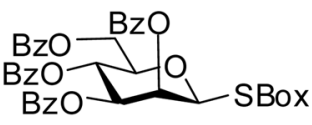

4

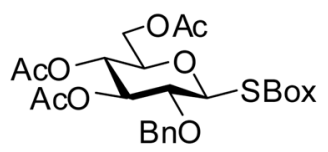

7

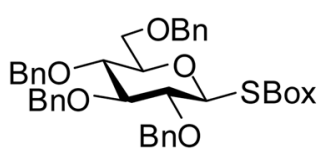

5

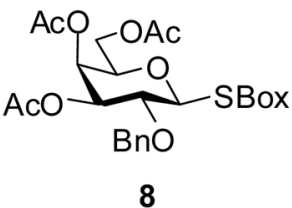

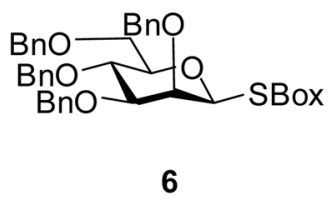

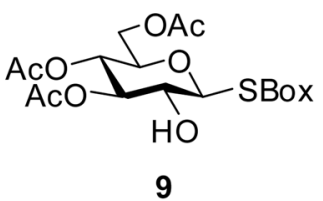

Figure 1.

Differently protected SBox glycosides of the D-gluco, D-galacto, and D-manno series 


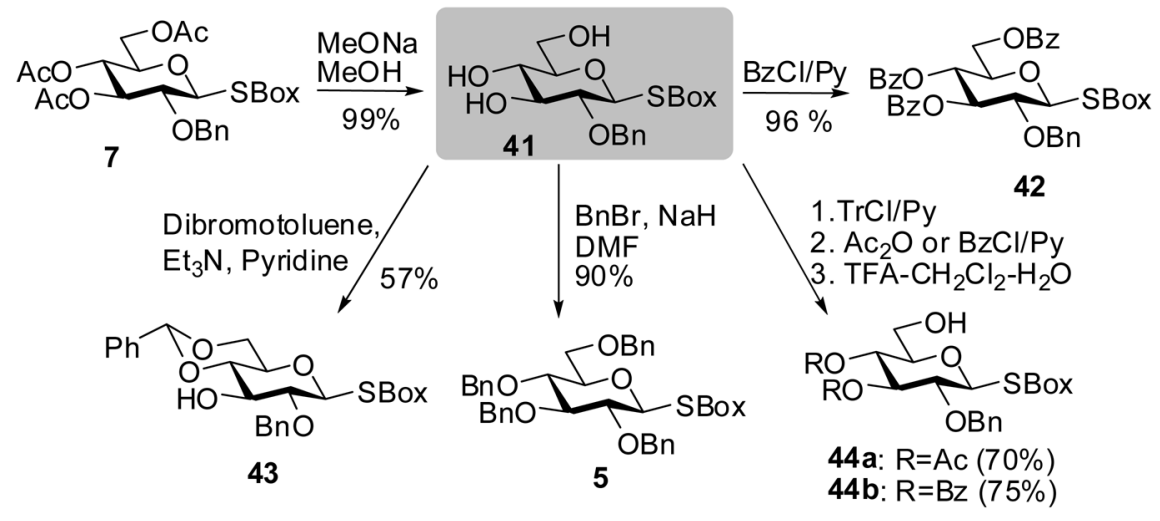

Scheme 1. 


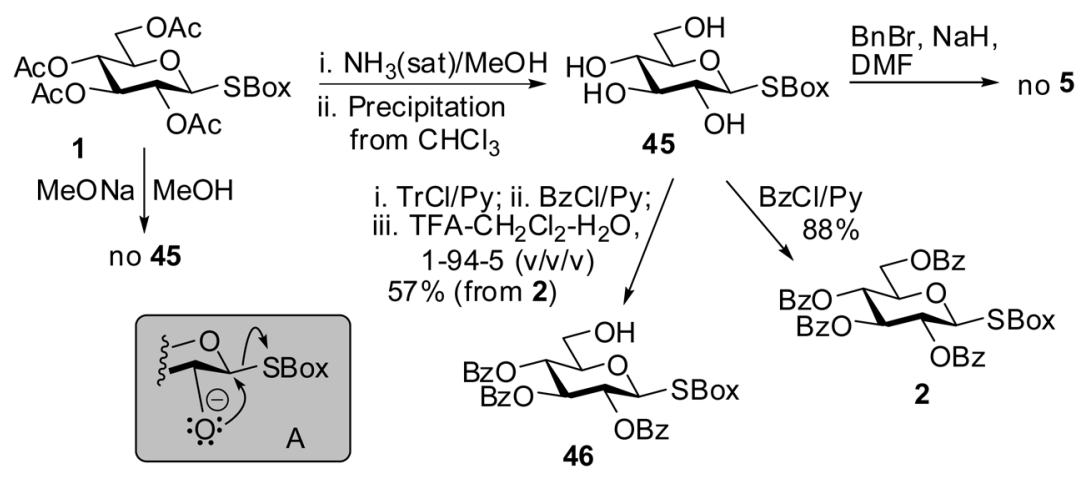

Scheme 2. 


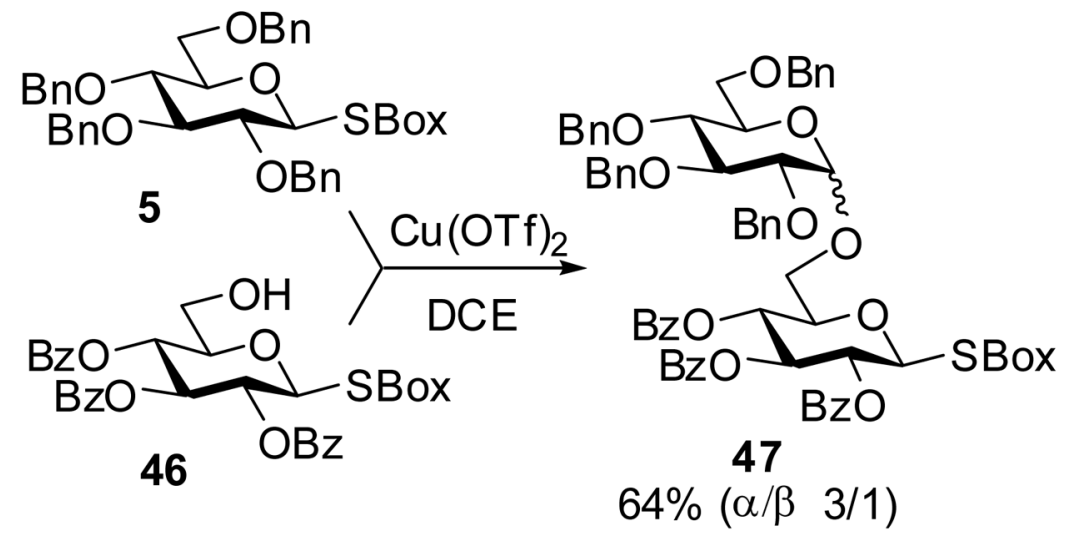

Scheme 3. 


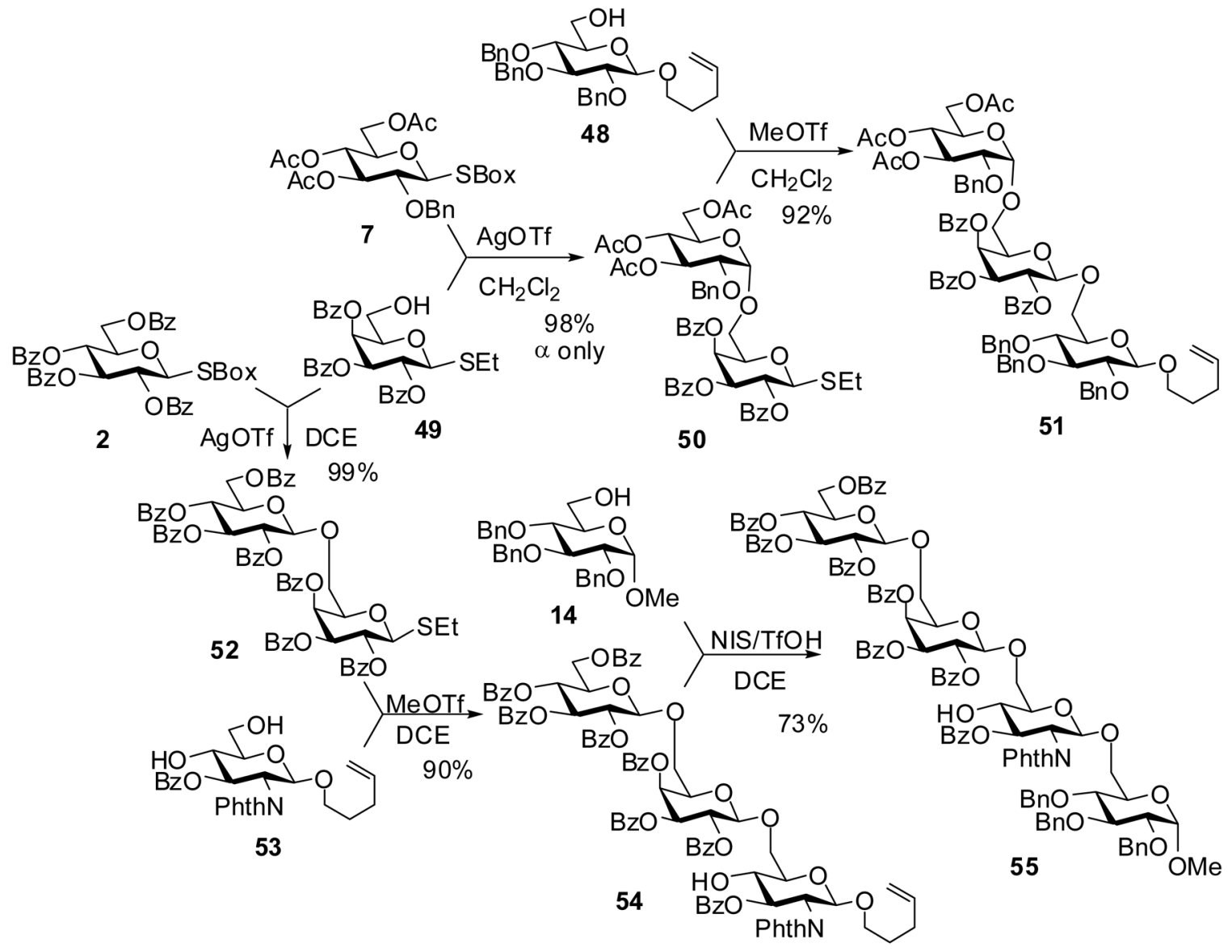

Scheme 4. 


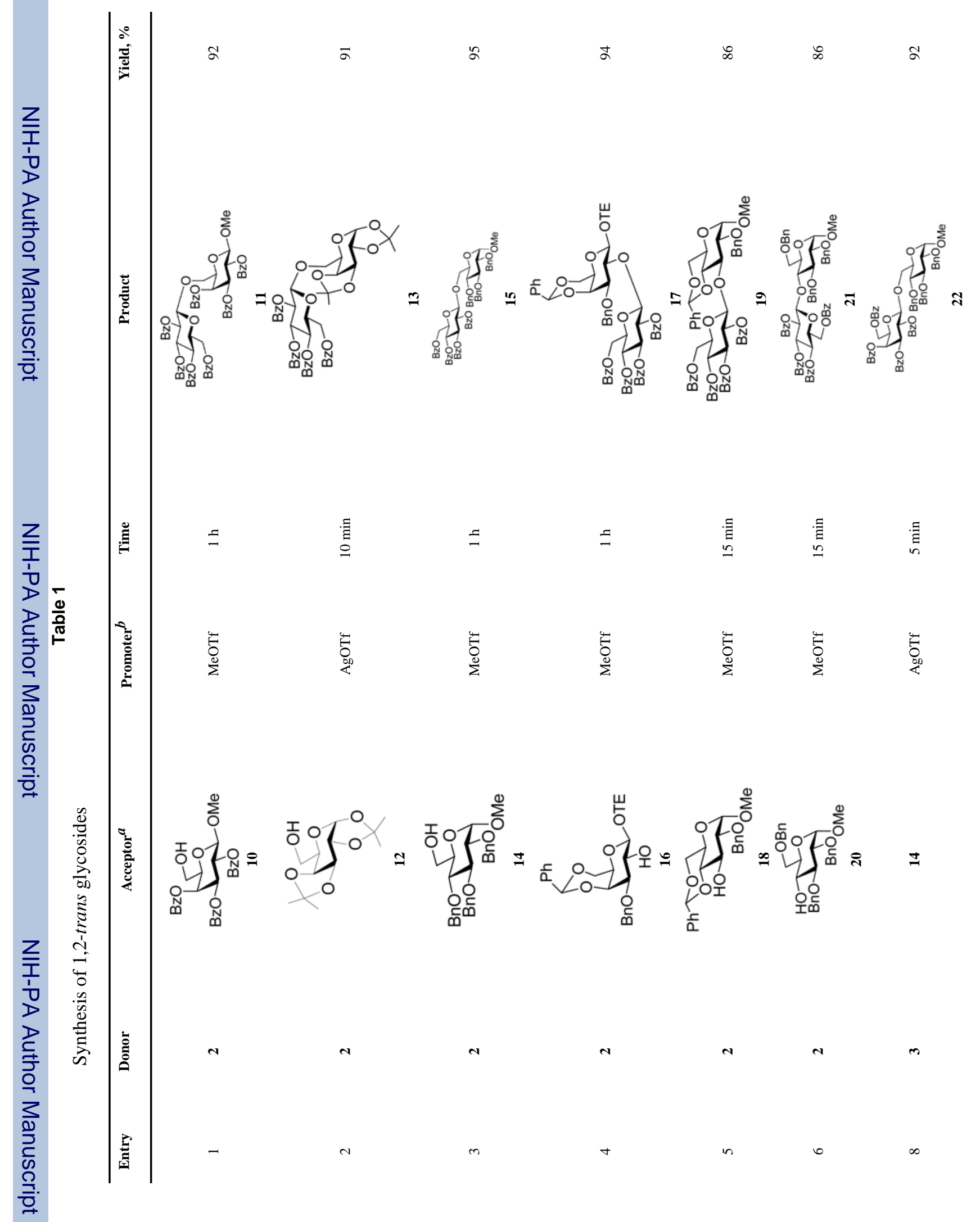




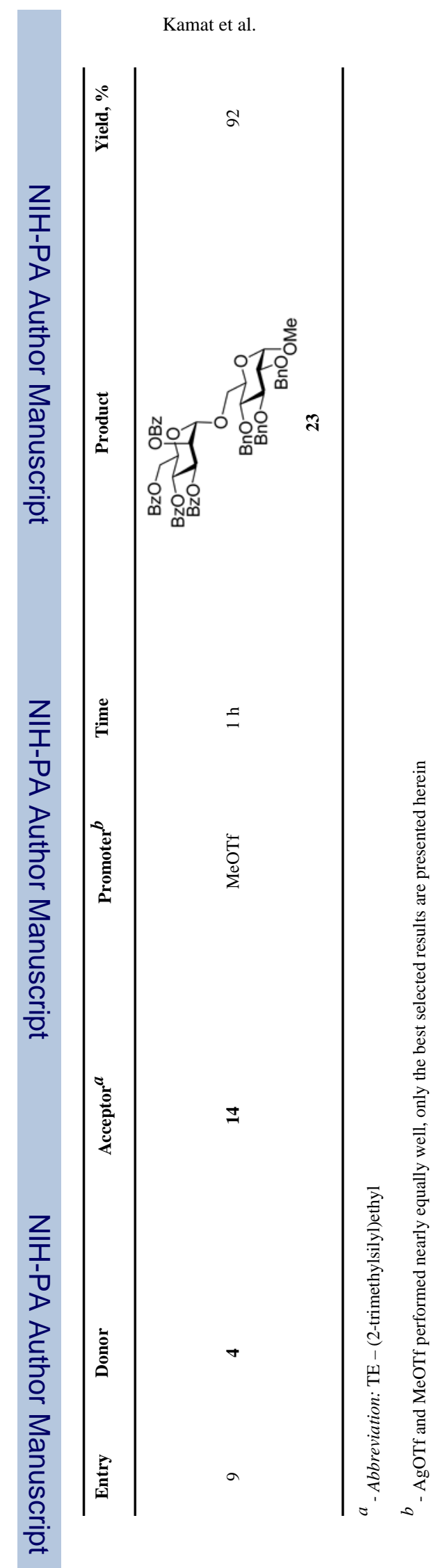

J Org Chem. Author manuscript; available in PMC 2008 September 12. 


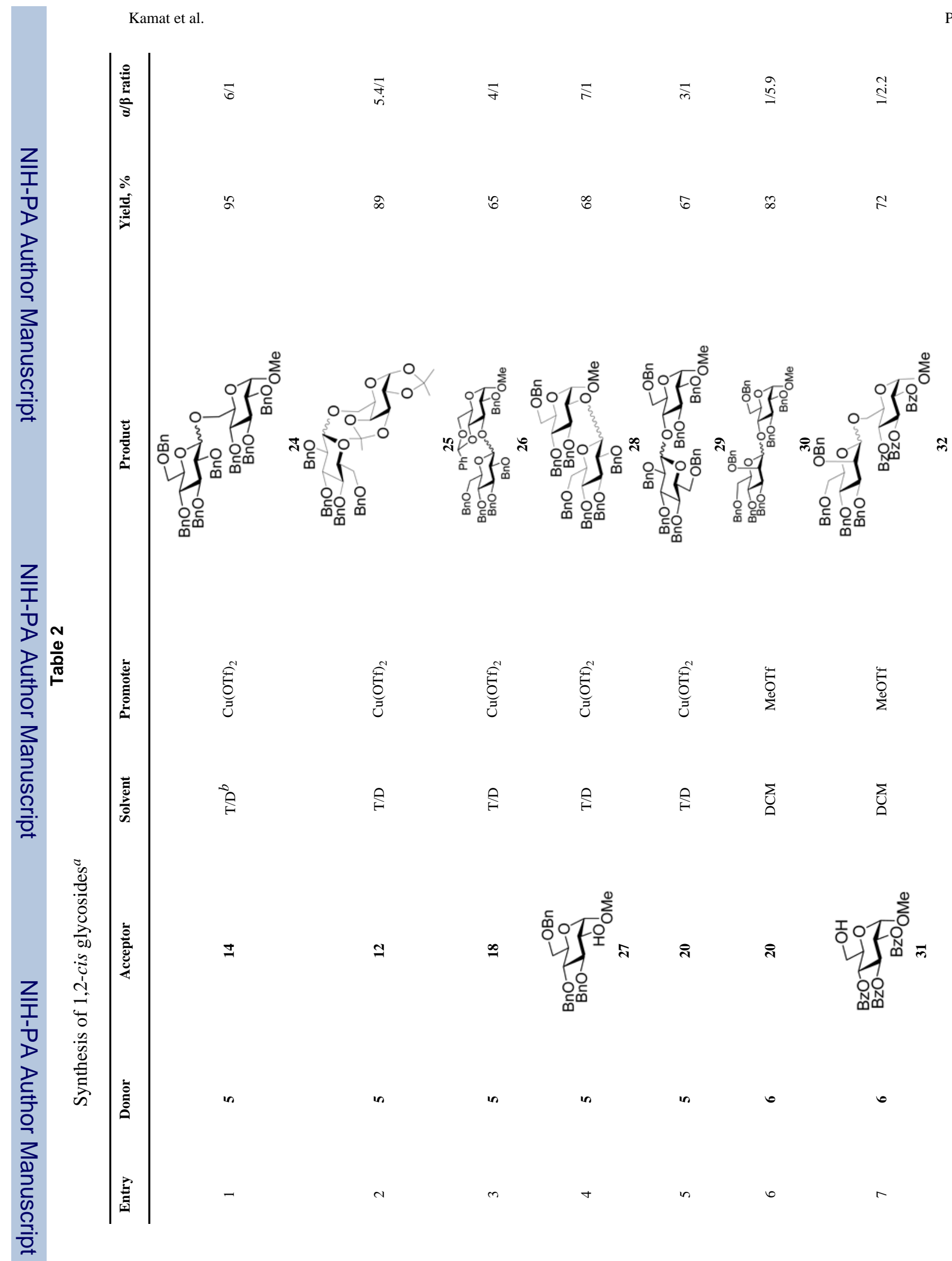

J Org Chem. Author manuscript; available in PMC 2008 September 12. 


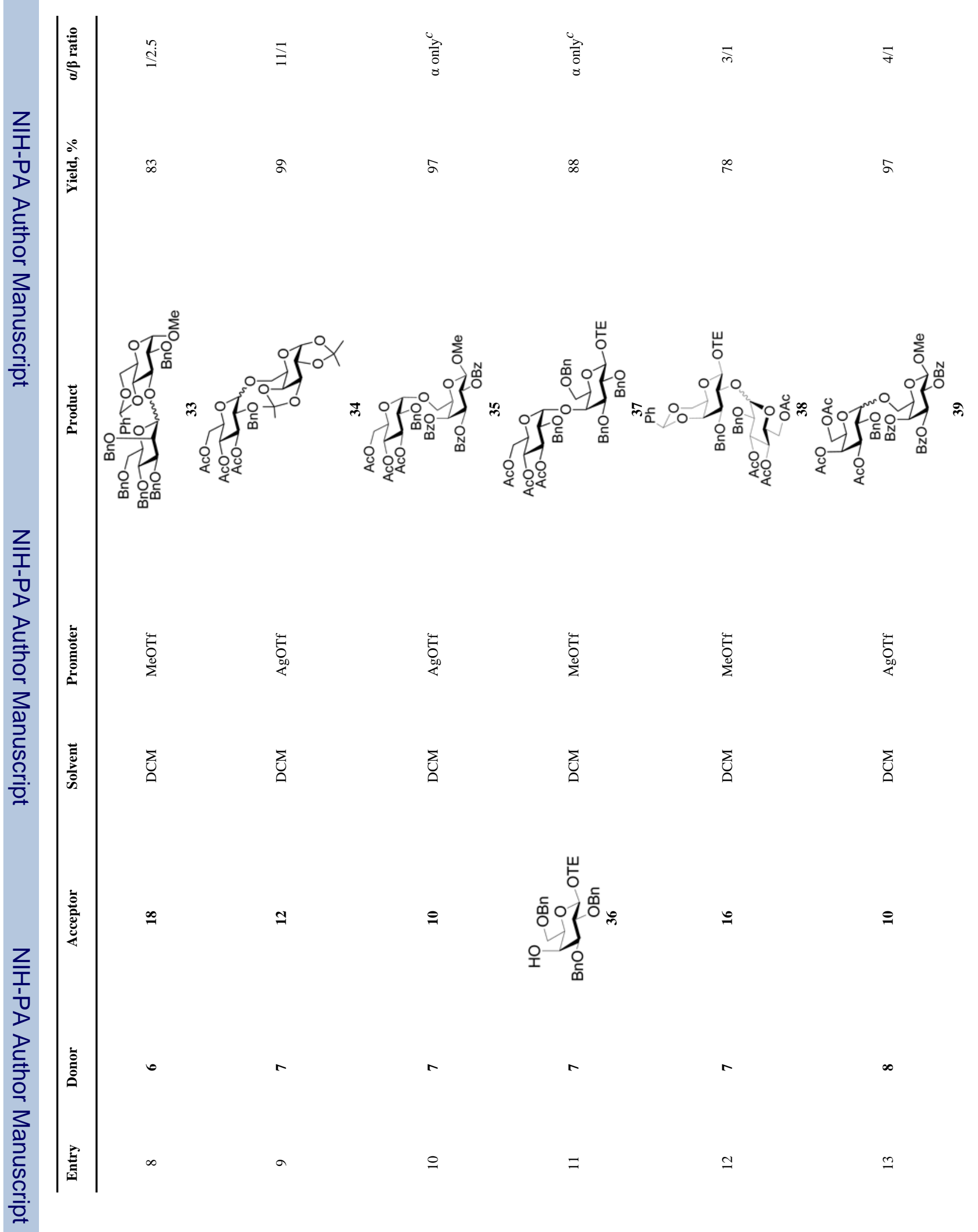




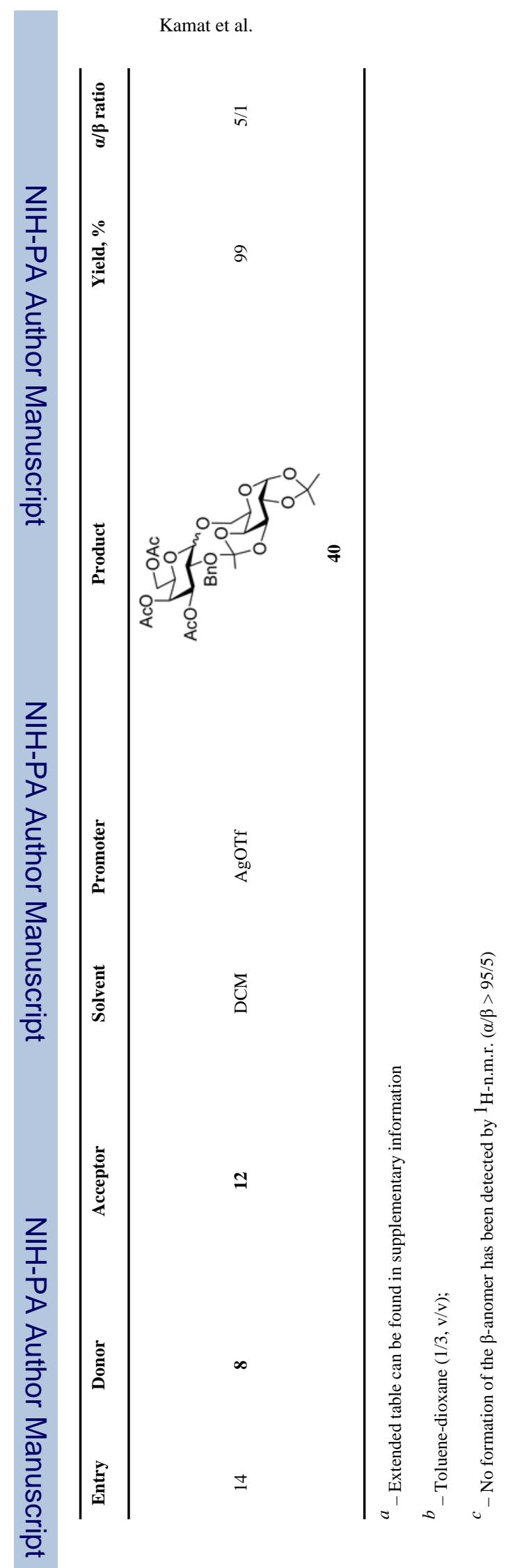

J Org Chem. Author manuscript; available in PMC 2008 September 12. 